\title{
Health care use before a diagnosis of primary intracranial tumor: a Danish nationwide register study
}

This article was published in the following Dove Press journal:

Clinical Epidemiology

\author{
Charlotte Nygaard' \\ Henry Jensen' \\ Jakob Christensen ${ }^{2,3}$ \\ Peter Vedsted ${ }^{1,4}$ \\ 'Research Centre for Cancer \\ Diagnosis in Primary Care (CaP), \\ Research Unit for General Practice \\ \& Section for General Medicine, \\ Department of Public Health, \\ Aarhus University, Aarhus, Denmark; \\ ${ }^{2}$ Department of Clinical Medicine, \\ Aarhus University, Aarhus, Denmark; \\ ${ }^{3}$ Department of Neurology, Aarhus \\ University Hospital, Aarhus, Denmark; \\ ${ }^{4}$ Department of Clinical Medicine, \\ Aarhus University \& Diagnostic \\ Center, University Research Clinic for \\ Innovative Patient Pathways, Silkeborg \\ Regional Hospital, Silkeborg, Denmark
}

Correspondence: Charlotte Nygaard Research Centre for Cancer Diagnosis in Primary Care (CaP), Research Unit for General Practice \& Section for General Medicine, Department of Public Health, Aarhus University, Bartholins Allé 2, DK-8000 Aarhus C, Denmark

Tel +45 4242 8I 45

Email charlotte_nygaard@hotmail.com
Introduction: Detailed knowledge of prediagnostic health care use among patients with primary intracranial tumors is sparse. We aimed to investigate the health care use among adults during the 2 years preceding a diagnosis of a benign or malignant primary intracranial tumor in Denmark.

Methods: We conducted a population-based matched cohort study using historical data from Danish nationwide registries, including all patients aged 30-90 years diagnosed with a first-time primary intracranial tumor from January 1, 2009 to December 31, 2014, and with no prior cancer diagnosis $(n=5,926)$. For each patient, ten comparison subjects were identified using density sampling. We analyzed differences in the frequency and timing of health care use within general practice, physiotherapy, radiology, ear-nose-throat, ophthalmology, neurology, and psychiatry. Odds ratios of having multiple contacts were calculated using a conditional logistical regression model. Monthly incidence rate ratios were estimated using a negative binomial regression model. Results: Of all patients, $62 \%$ had a benign tumor. Patients with benign tumors were more likely to have multiple consultations with health care providers in the period 2-12 months prior to diagnosis and to have increased rates of consultations 1-24 months prior to diagnosis, depending on health service.

Conclusion: Patients diagnosed with a benign or a malignant primary intracranial tumor use the health care services differently. Increased health care use is seen within relatively close proximity to the diagnosis for patients with malignant tumors. However, patients with benign tumors have increased health care use from up to 2 years prior to diagnosis; this suggests a window of opportunity for earlier diagnosis.

Keywords: general practice, diagnosis, brain neoplasms, health care services, Denmark

\section{Introduction}

Early diagnosis of primary intracranial tumors is important as some of them, eg, meningiomas, can be radically resected. Additionally, low-grade astrocytomas may transform into high-grade astrocytomas. ${ }^{1}$ More than half of the primary intracranial tumors among adults are malignant astrocytoma and meningioma. ${ }^{2}$ The prognosis varies from a 5 -year survival of glioblastoma of $<5 \%$ to a 10 -year survival of meningioma of $90 \%{ }^{2}$

General practitioners (GPs) are often involved in the diagnosis, particularly in the very early phases of the disease. ${ }^{3,4}$ With approximately 1,500 primary intracranial tumor patients annually in Denmark, ${ }^{5}$ a Danish GP will on average see 1 patient with an intracranial tumor every third year. Identifying patients in general practice who are suspected of having a primary intracranial tumor is a difficult task as symptoms 
of cancer generally have low positive predictive values in general practice. ${ }^{6}$

Primary intracranial tumors often present with specific focal neurological deficits, such as loss of motor function, sensory loss, abnormalities of the visual fields, or with newonset seizure. ${ }^{7}$ Nevertheless, many symptoms of intracranial tumors are vague and nonspecific, such as headache, nausea, dizziness, confusion, anxiety, depression, fatigue, and memory loss; these are symptoms that mostly are caused by other conditions apart from intracranial tumors. ${ }^{8}$ All symptoms of brain tumors, except new-onset epilepsy, are individually linked with low risk in general practice. ${ }^{7}$ Thus, primary intracranial tumors possess important features seen with prolonged cancer diagnostics in general: low incidence and uncharacteristic symptoms. ${ }^{9}$ Delayed diagnosis may have important consequences for the patients in terms of higher mortality and morbidity and more advanced stages of disease at treatment initiation. ${ }^{10}$

Although increased health care use can be a proxy for symptom presentation and how health care professionals act on this symptom presentation, ${ }^{11}$ little is known about how and when adults seek health care before a primary intracranial tumor diagnosis. The sparse evidence indicates that a high proportion of patients with a brain tumor has multiple primary care consultations before referral ${ }^{12}$ and that children and young patients with central nervous system (CNS) tumors have elevated health care use from 17 months prior to diagnosis. ${ }^{11}$

We aimed to investigate the health care use among adults during the 2 years preceding a diagnosis of a benign or a malignant primary intracranial tumor in Denmark.

\section{Methods}

We performed a population-based matched comparative study using national registry data. Data was linked at the individual level by using the Danish civil registration number assigned to all Danish citizens. ${ }^{13}$

\section{Setting}

The publicly funded health care system in Demark provides free access for all citizens to general practice and hospital care, and nearly all citizens (98\%) are registered with a GP. ${ }^{14}$ The GPs are gatekeepers to the rest of the health care system as they carry out initial diagnostic investigations and refer patients to hospitals or outpatient clinics if needed. ${ }^{15}$ Patients may, however, contact an ear-nosethroat (ENT) specialist or an ophthalmologist without referral from a GP. ${ }^{15}$

\section{Study population}

Patients were identified in the Danish Cancer Registry ${ }^{16}$ and were eligible if diagnosed with a first-time primary intracranial tumor, benign (International Classification of Diseases, 10th revision [ICD-10] ${ }^{17}$ : D320, D330-332, D333B, D333A, D352*, D353, D354) or malignant (ICD-10: C722, C723, C724, C725, C751, C752, C753, C700, C71*), from January 1, 2009 to December 31, 2014, and aged 30-90 years at the date of diagnosis. In total, 6,956 patients were identified.

We excluded 1,030 (14.8\%) patients: 822 (11.8\%) due to a previous or concurrent cancer (previous nonmelanoma skin cancer [C44] did not result in exclusion), 30 (0.4\%) due to no registration at Statistics Denmark, 94 (1.4\%) who lived outside Denmark at some point during the 24 months preceding diagnosis, and 84 (1.2\%) who were not listed with a general practice.

The study was approved by the Danish Data Protection Agency (file no. 2009-41-3471). According to Danish law, the study did not require approval from the Committee on Health Research Ethics of the Central Denmark Region as no biomedical intervention was performed.

\section{Comparison subjects}

Using incidence density sampling, we identified 10 comparison subjects matched on age, gender, and listed general practice for each patient. Information about general practice affiliation was available from the Patient List Register, which holds information on persons assigned to a certain GP at any given time. The index date was defined as the date of the diagnosis of the corresponding patient.

Comparison subjects were not eligible if they fulfilled any of the above listed exclusion criteria for patients. The age difference between patient and the corresponding comparison subject exceeded 2 years for $489(0.8 \%)$ comparison subjects. They were replaced by comparison subjects sampled among patients listed with random general practices in Denmark.

\section{Outcomes}

The main outcomes of the study were daytime face-to-face consultations in general practice, including home visits, physiotherapy consultations, radiological examinations, and face-to-face contact with the following specialties: ENT, ophthalmology, neurology, and psychiatry, and all other hospital contacts.

For radiology contacts, we included all types of overall computed tomography, magnetic resonance, X-ray, and ultrasound scans made in primary or secondary care. This approach ensured that all examinations were counted 
regardless of the organizational place of the radiological examination, thus making it possible to compare patients with primary intracranial tumors with comparison subjects prior to diagnosis. Data regarding consultations in primary care were extracted from the Danish National Health Service Register, ${ }^{18}$ which holds information on all services provided by general practice, physiotherapists, private specialist doctors, and radiological examinations at private radiological clinics for the purpose of remuneration.

Data regarding contacts to somatic hospital (inpatient and outpatient contacts) and data on radiological examinations in secondary health care were extracted from the Danish National Patient Register (DNPR), which contains information on all in- and outpatient contacts at somatic hospitals in Denmark since 1977; outpatient contacts have been included in the register since 1995. ${ }^{19}$ Diagnostic information in DNPR is based on the ICD, 8th revision from 1977 to 1993 , and the ICD-10 from 1994 to $2016 .{ }^{19}$ Information on psychiatric inpatient and outpatient contacts was retrieved from the DanishPsychiatric Central Research Register, which holds information on all patient contacts with psychiatric departments in Denmark. ${ }^{20}$

To investigate the total consultation pattern across medical specialties, the measures of radiological examination, ENT, ophthalmology, neurology, and psychiatry represent the sum of contacts in both primary and secondary health care.

\section{Covariates}

The covariates included were age, comorbidity, marital status, income, educational level, labor market affiliation, and country of origin. Additionally, information on known diseases within 4 medical specialties of interest (ENT, ophthalmology, neurology, and psychiatry) was used for subanalyses.

Age was defined as the age at the index date. To adjust for comorbidity, the Charlson Comorbidity Index (CCI) was calculated $^{21}$ based on diagnoses registered in the DNPR 10 years prior to the study entry ( 2 years prior to the index date) and grouped into "None" (CCI score $=0$ ), "Moderate" (CCI score $=1-2$ ), and "Severe" (CCI score $\geq 3$ ).

Demographic and socioeconomic information was collected from Statistics Denmark. ${ }^{22}$ Marital status was categorized into "Living alone" or "Married/Cohabitating." Data on taxable income was extracted for the calendar year preceding the index date to eliminate the risk of influence from the primary intracranial tumor diagnosis. Income was subdivided into 3 equally sized groups using the OECDmodified scale and classified as "Low," "Middle", or "High" income. ${ }^{23}$ The highest attained level of education was categorized into "Basic," "Short", and "Long" according to the
International Standard Classification of Education. ${ }^{24}$ Labor market affiliation based on the main employment during the preceding 12 months was categorized into "Working," "Unemployed" (including maternity leave and unspecified leave), and "Retired" (including persons on early retirement and persons receiving early retirement benefit). Country of origin was categorized as "Danish" or "Immigrant."

Persons with missing information on marital status $(n=1)$ were categorized as "Cohabiting." Persons with missing information on income $(n=1)$ were categorized as "Low income." Persons with missing information on education (357 patients, 3,854 comparison subjects) were categorized as "Basic education," as these persons are most often uneducated. ${ }^{25}$ Persons with missing information on labor market affiliation (200 patients, 2,009 comparison subjects) were categorized as "Unemployed," as these persons most often have no income, ${ }^{26}$ and persons with missing information on nationality $(n=1)$ were categorized as "Danish."

Data on known diseases within the 4 specific medical specialties ENT, ophthalmology, neurology, and psychiatry, were retrieved from the DNPR according to medical specialty-specific ICD-10 codes, except for diagnoses already accounted for in the CCI. The known diseases of interest were defined as ENT: "diseases in ear and mastoid process of temporal bone (DH60-DH95);" ophthalmology: "diseases in eye and orbital region (DH00-DH59);" neurology: "diseases in the nervous system (G00-G29, G31-G44, G46-G79, and G83-G99);" and psychiatry: "psychiatric disease and behavioral disorders (F03-F05.0 and F05.2-F99)."

\section{Statistical analysis}

Analyses were stratified on whether patients were diagnosed with a benign or a malignant primary intracranial tumor. The analyses were performed for each sex separately because of known gender differences in the health care utilization. ${ }^{27}$

Odds ratios (ORs) of having consultations in the year prior to diagnosis (omitting the last month before diagnosis) were estimated using conditional logistical regression to account for the matched design.

A negative binominal regression model applying cluster robust variance at the patient level was used to estimate incidence rate ratios (IRRs) for comparison of the monthly rates of health care contacts between patients and comparison subjects in the entire 2-year period before diagnosis.

Analyses were adjusted for age, comorbidity, marital status, income, educational level, labor market affiliation, and country of origin. 
To investigate if known diseases within a medical specialty influenced the estimates, we undertook subanalyses on the ORs of having a consultation at ENT, ophthalmology, neurology, and psychiatry, by adjusting for the presence of known disease of the specific medical specialty in question (ie, the ORs of having a consultation in ophthalmology was also adjusted for known diseases in the eye and orbital region).

Data were analyzed using the statistical software Stata 14 (StataCorp LP, College Station, TX, USA).

\section{Results}

The study included 5,926 incident first-time primary intracranial tumor patients and 59,260 comparison subjects. Of the patients identified 3,654 (62\%) had a benign tumor ( $70 \%$ among women and $52 \%$ among men, respectively).
The patients and the comparison subjects were generally comparable, although more patients with benign tumors than their comparison subjects had comorbidity (Table 1). The opposite trend was observed for patients with malignant tumors.

\section{Patients with benign primary intracranial tumors}

In the year preceding diagnosis (omitting the last month before diagnosis), the proportion of female patients diagnosed with a benign tumor who consulted the different health care providers were as follows: GP: $91.1 \%$, physiotherapy: 14.1\%, radiology: $47.2 \%$, ENT: $19.2 \%$, ophthalmology: $24.0 \%$, neurology: $7.4 \%$, psychiatry: $3.6 \%$, and other hospital contacts: $45.6 \%$ (Table 2 ). The numbers were slightly lower for men.

Table I Characteristics of the study population of patients with benign or malignant primary intracranial tumors diagnosed in 20092014 stratified on gender

\begin{tabular}{|c|c|c|c|c|c|c|c|c|c|c|c|c|}
\hline \multicolumn{13}{|l|}{ Benign tumors } \\
\hline & \multicolumn{6}{|c|}{ Women } & \multicolumn{6}{|c|}{ Men } \\
\hline & \multicolumn{2}{|c|}{$\begin{array}{l}\text { Comparison } \\
\text { subjects }\end{array}$} & \multicolumn{2}{|c|}{ Patients } & \multicolumn{2}{|l|}{ Total } & \multicolumn{2}{|c|}{$\begin{array}{l}\text { Comparison } \\
\text { subjects }\end{array}$} & \multicolumn{2}{|c|}{ Patients } & \multicolumn{2}{|l|}{ Total } \\
\hline & $\mathbf{n}$ & $\%$ & $\mathbf{n}$ & $\%$ & $\mathbf{n}$ & $\%$ & $\mathbf{n}$ & $\%$ & $\mathbf{n}$ & $\%$ & $\mathbf{n}$ & $\%$ \\
\hline & $\overline{22,160}$ & $\overline{90.9}$ & $\overline{2,216}$ & 9.1 & $\overline{24,376}$ & 100 & 14,380 & 90.9 & $\overline{1,438}$ & $\overline{9.1}$ & $\overline{15,818}$ & 100 \\
\hline \multicolumn{13}{|l|}{ Age group (years) } \\
\hline $30-49$ & 6,090 & 27.5 & 609 & 27.5 & 6,699 & 27.5 & 3,920 & 27.3 & 392 & 27.3 & 4,312 & 27.3 \\
\hline $50-69$ & 10,350 & 46.7 & 1,035 & 46.7 & 11,385 & 46.7 & 7,210 & 50.1 & 721 & 50.1 & 7,931 & 50.1 \\
\hline 70-90 & 5,720 & 25.8 & 572 & 25.8 & 6,292 & 25.8 & 3,250 & 22.6 & 325 & 22.6 & 3,575 & 22.6 \\
\hline \multicolumn{13}{|l|}{ Country of origin } \\
\hline Danish & 20,445 & 92.3 & 2,058 & 92.9 & 22,503 & 92.3 & $13,3 \mid 3$ & 92.6 & I,342 & 93.3 & 14,655 & 92.6 \\
\hline Immigrant & 1,715 & 7.7 & 158 & 7.1 & 1,873 & 7.7 & 1,067 & 7.4 & 96 & 6.7 & 1,163 & 7.4 \\
\hline \multicolumn{13}{|l|}{ Marital status } \\
\hline Cohabiting & 13,915 & 62.8 & 1,390 & 62.7 & 15,305 & 62.8 & 10,384 & 72.2 & $\mathrm{I}, 054$ & 73.3 & 11,438 & 72.3 \\
\hline Living alone & 8,245 & 37.2 & 826 & 37.3 & $9,07 \mid$ & 37.2 & 3,996 & 27.8 & 384 & 26.7 & 4,380 & 27.7 \\
\hline \multicolumn{13}{|l|}{ Educational level } \\
\hline Basic & 7,907 & 35.7 & 779 & 35.2 & 8,686 & 35.6 & 4,255 & 29.6 & 428 & 29.8 & 4,683 & 29.6 \\
\hline Short & 8,069 & 36.4 & 814 & 36.7 & 8,883 & 36.4 & 6,543 & 45.5 & 633 & 44.0 & 7,176 & 45.4 \\
\hline Long & 6,184 & 27.9 & 623 & 28.1 & 6,807 & 27.9 & 3,582 & 24.9 & 377 & 26.2 & 3,959 & 25.0 \\
\hline \multicolumn{13}{|l|}{ Labor market affiliation } \\
\hline Working & 10,453 & 47.2 & 992 & 44.8 & $\mathrm{II}, 445$ & 47.0 & 7,718 & 53.7 & 756 & 52.6 & 8,474 & 53.6 \\
\hline Unemployed & 1,384 & 6.2 & 153 & 6.9 & $\mathrm{I}, 537$ & 6.3 & 757 & 5.3 & 84 & 5.8 & 841 & 5.3 \\
\hline Retirement pension & 10,323 & 46.6 & $|, 07|$ & 48.3 & 11,394 & 46.7 & 5,905 & 41.1 & 598 & 41.6 & 6,503 & 41.1 \\
\hline \multicolumn{13}{|l|}{ Income } \\
\hline Low & 7,477 & 33.7 & 759 & 34.3 & 8,236 & 33.8 & 4,132 & 28.7 & 416 & 28.9 & 4,548 & 28.8 \\
\hline Middle & 7,476 & 33.7 & 736 & 33.2 & 8,212 & 33.7 & 4,855 & 33.8 & 481 & 33.4 & 5,336 & 33.7 \\
\hline High & 7,207 & 32.5 & 721 & 32.5 & 7,928 & 32.5 & 5,393 & 37.5 & 541 & 37.6 & 5,934 & 37.5 \\
\hline \multicolumn{13}{|l|}{ Comorbidity } \\
\hline Low & 18,385 & 83.0 & I,786 & 80.6 & $20,|7|$ & 82.7 & 11,715 & 81.5 & $\mathrm{I}, 145$ & 79.6 & 12,860 & 81.3 \\
\hline Moderate & 2,486 & 11.2 & 274 & 12.4 & 2,760 & 11.3 & 1,625 & 11.3 & 179 & 12.4 & 1,804 & II.4 \\
\hline Severe & 1,289 & 5.8 & 156 & 7.0 & $\mathrm{I}, 445$ & 5.9 & $\mathrm{I}, 040$ & 7.2 & 114 & 7.9 & $\mathrm{I}, 154$ & 7.3 \\
\hline
\end{tabular}


Table I (Continued)

\begin{tabular}{|c|c|c|c|c|c|c|c|c|c|c|c|c|}
\hline \multicolumn{13}{|l|}{ Malignant tumors } \\
\hline & \multicolumn{6}{|c|}{ Women } & \multicolumn{6}{|c|}{ Men } \\
\hline & \multicolumn{2}{|c|}{$\begin{array}{l}\text { Comparison } \\
\text { subjects }\end{array}$} & \multicolumn{2}{|c|}{ Patients } & \multicolumn{2}{|l|}{ Total } & \multicolumn{2}{|c|}{$\begin{array}{l}\text { Comparison } \\
\text { subjects }\end{array}$} & \multicolumn{2}{|c|}{ Patients } & \multicolumn{2}{|l|}{ Total } \\
\hline & $n$ & $\%$ & $\mathbf{n}$ & $\%$ & $\mathbf{n}$ & $\%$ & $\mathbf{n}$ & $\%$ & $\mathbf{n}$ & $\%$ & $\mathbf{n}$ & $\%$ \\
\hline & 9,510 & 90.9 & 951 & 9.1 & $\overline{10,461}$ & 100 & $\overline{13,210}$ & 90.9 & $I, 32 I$ & 9.1 & $|4,53|$ & 100 \\
\hline \multicolumn{13}{|l|}{ Age group (years) } \\
\hline $30-49$ & 1,320 & 13.9 & 132 & 13.9 & $\mathrm{I}, 452$ & 13.9 & 2,140 & 16.2 & 214 & 16.2 & 2,354 & 16.2 \\
\hline $50-69$ & 4,690 & 49.3 & 469 & 49.3 & 5,159 & 49.3 & 7,170 & 54.3 & 717 & 54.3 & 7,887 & 54.3 \\
\hline $70-90$ & 3,500 & 36.8 & 350 & 36.8 & 3,850 & 36.8 & 3,900 & 29.5 & 390 & 29.5 & 4,290 & 29.5 \\
\hline \multicolumn{13}{|l|}{ Country of origin } \\
\hline Danish & 8,939 & 94.0 & 900 & 94.6 & 9,839 & 94.1 & 12,379 & 93.7 & $\mathrm{I}, 252$ & 94.8 & $|3,63|$ & 93.8 \\
\hline Immigrant & 571 & 6.0 & 51 & 5.4 & 622 & 5.9 & 831 & 6.3 & 69 & 5.2 & 900 & 6.2 \\
\hline \multicolumn{13}{|l|}{ Marital status } \\
\hline Cohabiting & 5,667 & 59.6 & 577 & 60.7 & 6,244 & 59.7 & 9,554 & 72.3 & 967 & 73.2 & $10,52 \mid$ & 72.4 \\
\hline Living alone & 3,843 & 40.4 & 374 & 39.3 & 4,217 & 40.3 & 3,656 & 27.7 & 354 & 26.8 & 4,010 & 27.6 \\
\hline \multicolumn{13}{|l|}{ Educational level } \\
\hline Basic & 3,940 & $4 I .4$ & 420 & 44.2 & 4,360 & 41.7 & 4,255 & 32.2 & 424 & 32.1 & 4,679 & 32.2 \\
\hline Short & 3,299 & 34.7 & 325 & 34.2 & 3,624 & 34.6 & 6,026 & 45.6 & 585 & 44.3 & 6,611 & 45.5 \\
\hline Long & 2,271 & 23.9 & 206 & 21.7 & 2,477 & 23.7 & 2,929 & 22.2 & 312 & 23.6 & 3,241 & 22.3 \\
\hline \multicolumn{13}{|l|}{ Labor market affiliation } \\
\hline Working & $3,34 I$ & 35.1 & 328 & 34.5 & 3,669 & 35.1 & 6,223 & 47.1 & 641 & 48.5 & 6,864 & 47.2 \\
\hline Unemployed & 419 & 4.4 & 42 & 4.4 & 461 & 4.4 & 589 & 4.5 & 50 & 3.8 & 639 & 4.4 \\
\hline Retirement pension & 5,750 & 60.5 & 581 & 61.1 & 6,331 & 60.5 & 6,398 & 48.4 & 630 & 47.7 & 7,028 & 48.4 \\
\hline \multicolumn{13}{|l|}{ Income } \\
\hline Low & 3,646 & 38.3 & 366 & 38.5 & 4,012 & 38.4 & 4,191 & 31.7 & 374 & 28.3 & 4,565 & 31.4 \\
\hline Middle & 3,095 & 32.5 & 317 & 33.3 & 3,412 & 32.6 & 4,394 & 33.3 & 462 & 35.0 & 4,856 & 33.4 \\
\hline High & 2,769 & 29.1 & 268 & 28.2 & 3,037 & 29.0 & 4,625 & 35.0 & 485 & 36.7 & 5,110 & 35.2 \\
\hline \multicolumn{13}{|l|}{ Comorbidity } \\
\hline Low & 7,594 & 79.9 & 779 & 81.9 & 8,373 & 80.0 & 10,306 & 78.0 & 1,070 & 81.0 & 11,376 & 78.3 \\
\hline Moderate & 1,215 & 12.8 & 116 & 12.2 & $|, 33|$ & 12.7 & $\mathrm{I}, 724$ & 13.1 & 158 & 12.0 & 1,882 & 13.0 \\
\hline Severe & 701 & 7.4 & 56 & 5.9 & 757 & 7.2 & 1,180 & 8.9 & 93 & 7.0 & $\mathrm{I}, 273$ & 8.8 \\
\hline
\end{tabular}

Patients with benign tumors had higher odds of having 5-9 consultations in general practice than comparison subjects (both sexes), and female patients had higher odds of having $\geq 10$ consultations in general practice than the comparison subjects (Table 2).

Overall, female and male patients had higher likelihood of having 1 or more contacts to ophthalmology, ENT, and neurology than the comparison subjects (Table 2). Female patients had a particularly higher likelihood of $\geq 3$ neurology contacts than the comparison subjects, which was not seen for male patients. Additionally, both male and female patients had higher odds of having 1 or more radiology contacts than the comparison subjects.

Overall, patients with benign tumors had significantly more consultations than the comparison subjects 1-24 months prior to diagnosis peeking the last month prior to diagnosis (Figures 1, 3, and S1). The starting point of increased health care use differed between male and female patients, eg, for general practice (men: 11 months, women: 20 months) and neurology (men: 9 months, women: 21 months). Further, the starting point of increased health care use differed across specialties, eg, ENT (both men and women: 24 months).

The peak in consultations 2 months prior to diagnosis ranged from $36 \%$ more consultations for physiotherapy $\left(\mathrm{IRR}_{\text {women }}=1.36[95 \%\right.$ confidence interval [CI]: $\left.1.08 ; 1.72]\right)$ to 19 times more consultations within neurology $\left(\mathrm{IRR}_{\text {women }}=9.61\right.$ [95\% CI: 15.83; 24.29]) (Figures 1 and 3).

\section{Patients with malignant primary intracranial tumors}

In the year preceding diagnosis (omitting the last month before diagnosis), the proportion of female patients diagnosed with a malignant tumor who consulted the different health care providers were as follows: GP: $87.1 \%$, physiotherapy: $13.5 \%$, radiology: $39.0 \%$, ENT: $14.2 \%$, 
Table 2 The associations (OR) between health care contacts and being diagnosed with a benign or malignant primary intracranial tumor (patients) or not (comparison subjects)

\begin{tabular}{|c|c|c|c|c|c|c|c|c|c|c|c|c|}
\hline \multicolumn{13}{|c|}{ Benign tumors } \\
\hline \multirow{3}{*}{$\begin{array}{l}\text { Number of } \\
\text { contacts by } \\
\text { specialty }\end{array}$} & \multicolumn{6}{|c|}{ Women } & \multicolumn{6}{|c|}{ Men } \\
\hline & \multicolumn{2}{|c|}{ Patients } & \multicolumn{2}{|c|}{$\begin{array}{l}\text { Comparison } \\
\text { subjects }\end{array}$} & \multirow[t]{2}{*}{ OR } & \multirow[t]{2}{*}{$95 \% \mathrm{Cl}$} & \multicolumn{2}{|c|}{ Patients } & \multicolumn{2}{|c|}{$\begin{array}{l}\text { Comparison } \\
\text { subjects }\end{array}$} & \multirow[t]{2}{*}{ OR } & \multirow[t]{2}{*}{$95 \% \mathrm{Cl}$} \\
\hline & $\mathbf{n}$ & $\%$ & $\mathbf{n}$ & $\%$ & & & $\mathbf{n}$ & $\%$ & $\mathbf{n}$ & $\%$ & & \\
\hline \multicolumn{13}{|l|}{ GP } \\
\hline 0 & 198 & 8.9 & 2,959 & 13.4 & 0.65 & $0.56-0.76$ & 239 & 16.6 & $3,4 I I$ & 23.7 & 0.64 & $0.55-0.74$ \\
\hline $1-4$ & 938 & 42.3 & 10,292 & 46.4 & 0.86 & $0.78-0.94$ & 704 & 49.0 & 6,840 & 47.6 & 1.07 & $0.95-1.19$ \\
\hline $5-9$ & 691 & 31.2 & 5,858 & 26.4 & 1.25 & $1.14-1.38$ & 334 & 23.2 & 2,721 & 18.9 & 1.30 & $1.14-1.49$ \\
\hline$\geq 10$ & 389 & 17.6 & 3,051 & 13.8 & 1.35 & $1.20-1.53$ & 161 & 11.2 & 1,408 & 9.8 & 1.14 & $0.94-1.37$ \\
\hline \multicolumn{13}{|c|}{ Physiotherapy (primary care) } \\
\hline 0 & 1,903 & 85.9 & 19,467 & 87.8 & 0.84 & $0.74-0.96$ & 1,308 & 91.0 & $|3,37|$ & 93.0 & 0.76 & $0.63-0.92$ \\
\hline $\mathrm{I}-3$ & 60 & 2.7 & 681 & 3.1 & 0.89 & $0.68-1.16$ & 38 & 2.6 & 323 & 2.2 & 1.17 & $0.83-1.65$ \\
\hline$\geq 4$ & 253 & 11.4 & 2,012 & 9.1 & 1.28 & $1.12-1.47$ & 92 & 6.4 & 686 & 4.8 & 1.37 & I.09-1.72 \\
\hline \multicolumn{13}{|c|}{ Radiology contacts } \\
\hline 0 & 1,169 & 52.8 & $|3,4| 4$ & 60.5 & 0.70 & $0.64-0.77$ & 948 & 65.9 & 10,940 & 76.1 & 0.60 & $0.53-0.67$ \\
\hline 1 & 445 & 20.1 & 3,724 & 16.8 & 1.27 & $1.13-1.44$ & 198 & 13.8 & $\mathrm{I}, 502$ & 10.4 & 1.36 & $1.15-1.60$ \\
\hline 2 & 204 & 9.2 & $\mathrm{I}, 834$ & 8.3 & 1.11 & $0.95-1.29$ & 123 & 8.6 & 760 & 5.3 & 1.68 & $1.37-2.05$ \\
\hline$\geq 3$ & 398 & 18.0 & 3,188 & 14.4 & 1.29 & I.14-I.45 & 169 & 11.8 & $\mathrm{I}, 178$ & 8.2 & 1.51 & $1.26-1.80$ \\
\hline \multicolumn{13}{|l|}{ ENT } \\
\hline 0 & I,79| & 80.8 & 19,677 & 88.8 & 0.52 & $0.47-0.59$ & $\mathrm{I}, 157$ & 80.5 & 12,826 & 89.2 & 0.49 & $0.42-0.57$ \\
\hline $\mathrm{I}-2$ & 279 & 12.6 & 1,816 & 8.2 & 1.63 & $1.42-1.87$ & 177 & 12.3 & $\mathrm{I}, 120$ & 7.8 & 1.67 & $1.4 \mid-1.98$ \\
\hline$\geq 3$ & 146 & 6.6 & 667 & 3.0 & 2.28 & $1.89-2.76$ & 104 & 7.2 & 434 & 3.0 & 2.52 & $2.01-3.16$ \\
\hline \multicolumn{13}{|c|}{ Ophthalmology } \\
\hline 0 & $\mathrm{I}, 684$ & 76.0 & $17,7 \mid 7$ & 80 & 0.79 & $0.7 I-0.88$ & 1,153 & 80.2 & 12,210 & 84.9 & 0.69 & $0.60-0.81$ \\
\hline $\mathrm{I}-2$ & 368 & 16.6 & 3,256 & 14.7 & 1.15 & $1.01-1.30$ & 215 & 15.0 & 1,593 & 11.1 & 1.45 & $1.23-1.70$ \\
\hline$\geq 3$ & 164 & 7.4 & $\mathrm{I}, 187$ & 5.4 & 1.42 & $1.19-1.70$ & 70 & 4.9 & 577 & 4.0 & 1.21 & $0.93-1.58$ \\
\hline \multicolumn{13}{|l|}{ Neurology } \\
\hline 0 & 2,053 & 92.6 & 21,299 & 96.1 & 0.53 & $0.45-0.64$ & 1,359 & 94.5 & 13,842 & 96.3 & 0.68 & $0.53-0.87$ \\
\hline $\mathrm{I}-2$ & 102 & 4.6 & 652 & 2.9 & 1.53 & $1.22-1.90$ & 59 & 4.1 & 395 & 2.7 & $\mathrm{I} .47$ & $1.11-1.96$ \\
\hline$\geq 3$ & 61 & 2.8 & 209 & 0.9 & 2.74 & $2.01-3.74$ & 20 & 1.4 & 143 & 1.0 & 1.51 & $0.94-2.43$ \\
\hline \multicolumn{13}{|l|}{ Psychiatry } \\
\hline 0 & 2,137 & 96.4 & 21,444 & 96.8 & 1.00 & $0.78-1.29$ & 1,394 & 96.9 & 14,056 & 97.7 & 0.71 & $0.48-1.03$ \\
\hline $\mathrm{I}-2$ & 19 & 0.9 & 216 & 1.0 & 0.74 & $0.44-1.22$ & 19 & 1.3 & 100 & 0.7 & 1.91 & $1.07-3.39$ \\
\hline$\geq 3$ & 60 & 2.7 & 500 & 2.3 & 1.12 & $0.83-|.5|$ & 25 & 1.7 & 224 & 1.6 & 1.20 & $0.74-1.93$ \\
\hline \multicolumn{13}{|c|}{ Other hospital specialties } \\
\hline 0 & 1,205 & 54.4 & 13,985 & 63.1 & 0.70 & $0.64-0.77$ & 837 & 58.2 & 9,697 & 67.4 & 0.67 & $0.59-0.75$ \\
\hline $\mathrm{I}-2$ & 499 & 22.5 & 4,054 & 18.3 & 1.29 & $1.16-1.44$ & 300 & 20.9 & 2,580 & 17.9 & 1.20 & $1.04-1.37$ \\
\hline$\geq 3$ & 512 & 23.1 & 4,121 & 18.6 & 1.29 & $1.16-1.44$ & 301 & 20.9 & 2,103 & 14.6 & 1.57 & $1.37-1.81$ \\
\hline
\end{tabular}

Malignant tumors

\begin{tabular}{|c|c|c|c|c|c|c|c|c|c|c|c|c|}
\hline \multirow{3}{*}{$\begin{array}{l}\text { Number of } \\
\text { contacts by } \\
\text { specialty }\end{array}$} & \multicolumn{6}{|c|}{ Women } & \multicolumn{6}{|c|}{ Men } \\
\hline & \multicolumn{2}{|c|}{ Patients } & \multicolumn{2}{|c|}{$\begin{array}{l}\text { Comparison } \\
\text { subjects }\end{array}$} & \multirow[t]{2}{*}{ OR } & \multirow[t]{2}{*}{$95 \% \mathrm{Cl}$} & \multicolumn{2}{|c|}{ Patients } & \multicolumn{2}{|c|}{$\begin{array}{l}\text { Comparison } \\
\text { subjects }\end{array}$} & \multirow[t]{2}{*}{ OR } & \multirow[t]{2}{*}{$95 \% \mathrm{Cl}$} \\
\hline & $\mathrm{n}$ & $\%$ & $\mathrm{n}$ & $\%$ & & & $\mathbf{n}$ & $\%$ & $n$ & $\%$ & & \\
\hline \multicolumn{13}{|l|}{ GP } \\
\hline 0 & 123 & 12.9 & 1,222 & 12.8 & 1.00 & $0.8 \mathrm{I}-\mathrm{I} .23$ & 293 & 22.2 & 2,836 & 21.5 & 1.03 & $0.89-1.19$ \\
\hline $1-4$ & 427 & 44.9 & $4,24 I$ & 44.6 & 1.01 & $0.88-1.16$ & 607 & 46.0 & 6,022 & 45.6 & 0.99 & $0.88-1.12$ \\
\hline $5-9$ & 271 & 28.5 & 2,619 & 27.5 & 1.05 & $0.91-1.22$ & 295 & 22.3 & 2,819 & 21.3 & 1.09 & $0.95-1.26$ \\
\hline$\geq 10$ & 130 & 13.7 & I,428 & 15.0 & 0.90 & $0.74-1.11$ & 126 & 9.5 & I,533 & 11.6 & 0.85 & $0.70-1.05$ \\
\hline \multicolumn{13}{|c|}{ Physiotherapy (primary care) } \\
\hline 0 & 823 & 86.5 & 8,346 & 87.8 & 0.88 & $0.72-1.08$ & 1,226 & 92.8 & 12,295 & 93.1 & 0.98 & $0.79-1.22$ \\
\hline $1-3$ & 28 & 2.9 & 257 & 2.7 & 1.12 & $0.75-1.68$ & 31 & 2.3 & 267 & 2.0 & 1.13 & $0.77-1.66$ \\
\hline$\geq 4$ & 100 & 10.5 & 907 & 9.5 & 1.13 & $0.90-1.4 \mid$ & 64 & 4.8 & 648 & 4.9 & 0.97 & $0.75-1.26$ \\
\hline
\end{tabular}


Table 2 (Continued)

\begin{tabular}{|c|c|c|c|c|c|c|c|c|c|c|c|c|}
\hline \multicolumn{13}{|c|}{ Malignant tumors } \\
\hline \multirow{3}{*}{$\begin{array}{l}\text { Number of } \\
\text { contacts by } \\
\text { specialty }\end{array}$} & \multicolumn{6}{|c|}{ Women } & \multicolumn{6}{|c|}{ Men } \\
\hline & \multicolumn{2}{|c|}{ Patients } & \multicolumn{2}{|c|}{$\begin{array}{l}\text { Comparison } \\
\text { subjects }\end{array}$} & \multirow[t]{2}{*}{ OR } & \multirow[t]{2}{*}{$95 \% \mathrm{Cl}$} & \multicolumn{2}{|c|}{ Patients } & \multicolumn{2}{|c|}{$\begin{array}{l}\text { Comparison } \\
\text { subjects }\end{array}$} & \multirow[t]{2}{*}{ OR } & \multirow[t]{2}{*}{$95 \% \mathrm{Cl}$} \\
\hline & $\mathbf{n}$ & $\%$ & $\bar{n}$ & $\%$ & & & $\bar{n}$ & $\%$ & $\bar{n}$ & $\%$ & & \\
\hline \multicolumn{13}{|c|}{ Radiology contacts } \\
\hline 0 & 580 & 61.0 & 5,794 & 60.9 & 0.99 & $0.85-1.14$ & 973 & 73.7 & 9,756 & 73.9 & 0.95 & $0.83-1.09$ \\
\hline 1 & 155 & 16.3 & $\mathrm{I}, 550$ & 16.3 & 1.01 & $0.83-1.22$ & 157 & 11.9 & 1,469 & II.I & 1.08 & $0.91-1.29$ \\
\hline 2 & 73 & 7.7 & 857 & 9.0 & 0.84 & $0.65-1.08$ & 74 & 5.6 & 749 & 5.7 & 1.00 & $0.78-1.29$ \\
\hline$\geq 3$ & 143 & 15.0 & 1,309 & 13.8 & 1.15 & $0.95-1.40$ & 117 & 8.9 & 1,236 & 9.4 & 1.01 & $0.82-1.25$ \\
\hline \multicolumn{13}{|l|}{ ENT } \\
\hline 0 & 816 & 85.8 & 8,315 & 87.4 & 0.87 & $0.7 I-1.06$ & 1,169 & 88.5 & $|I, 64|$ & 88.1 & 1.04 & $0.87-1.24$ \\
\hline $\mathrm{I}-2$ & 99 & 10.4 & 902 & 9.5 & 1.11 & $0.89-1.39$ & 103 & 7.8 & 1,121 & 8.5 & 0.91 & $0.73-1.12$ \\
\hline$\geq 3$ & 36 & 3.8 & 293 & 3.1 & 1.25 & $0.87-1.79$ & 49 & 3.7 & 448 & 3.4 & 1.12 & $0.82-1.52$ \\
\hline \multicolumn{13}{|c|}{ Ophthalmology } \\
\hline 0 & 727 & 76.4 & 7,357 & 77.4 & 0.94 & $0.80-1.11$ & 1,099 & 83.2 & 10,924 & 82.7 & 1.01 & $0.86-1.18$ \\
\hline $\mathrm{I}-2$ & 159 & 16.7 & I,547 & 16.3 & 1.04 & $0.87-1.25$ & 149 & 11.3 & 1,682 & 12.7 & 0.90 & $0.75-1.08$ \\
\hline$\geq 3$ & 65 & 6.8 & 606 & 6.4 & 1.09 & $0.83-1.43$ & 73 & 5.5 & 604 & 4.6 & 1.23 & $0.95-1.59$ \\
\hline \multicolumn{13}{|l|}{ Neurology } \\
\hline 0 & 903 & 95.0 & 9,123 & 95.9 & 0.81 & $0.60-1.10$ & 1,253 & 94.9 & 12,695 & 96.1 & 0.72 & $0.55-0.94$ \\
\hline $\mathrm{I}-2$ & 30 & 3.2 & 296 & 3.1 & 0.96 & $0.66-1.39$ & 54 & 4.1 & 377 & 2.9 & 1.51 & $1.12--2.03$ \\
\hline$\geq 3$ & 18 & 1.9 & 91 & 1.0 & 2.00 & $1.19-3.34$ & 14 & 1.1 & 138 & 1.0 & 1.08 & $0.62-1.89$ \\
\hline \multicolumn{13}{|l|}{ Psychiatry } \\
\hline 0 & 922 & 97.0 & 9,225 & 97 & 0.94 & $0.63-1.40$ & 1,297 & 98.2 & 12,936 & 97.9 & 1.03 & $0.66-1.59$ \\
\hline $\mathrm{I}-2$ & 7 & 0.7 & 94 & 1.0 & $0.7 \mathrm{I}$ & $0.31-1.61$ & $<5$ & - & 96 & 0.7 & 0.49 & $0.18-1.28$ \\
\hline$\geq 3$ & 22 & 2.3 & 191 & 2.0 & 1.25 & $0.80-1.95$ & 20 & 1.5 & 178 & 1.3 & 1.24 & $0.75-2.04$ \\
\hline \multicolumn{13}{|c|}{ Other hospital specialties } \\
\hline 0 & 621 & 65.3 & 5,959 & 62.7 & 1.09 & $0.94-1.27$ & 881 & 66.7 & 8,673 & 65.7 & 1.00 & $0.88-1.13$ \\
\hline $\mathrm{I}-2$ & 164 & 17.2 & ।,755 & 18.5 & 0.93 & $0.78-1.11$ & 227 & 17.2 & 2,308 & 17.5 & 0.99 & $0.85-1.15$ \\
\hline$\geq 3$ & 166 & 17.5 & 1,796 & 18.9 & 0.95 & $0.79-1.14$ & 213 & 16.1 & 2,229 & 16.9 & 1.03 & $0.87-1.21$ \\
\hline
\end{tabular}

Notes: Adjusted for country of origin, $\mathrm{CCl}$, marital status, income, labor market affiliation, highest educational level.

Abbreviations: $\mathrm{CCl}$, Charlson Comorbidity Index; Cl, confidence interval; ENT, ear-nose-throat; GP, general practitioner; OR, odds ratio.

ophthalmology: $23.6 \%$, neurology: 5.0\%, psychiatry: $3.0 \%$, and other hospital contacts: $34.7 \%$ (Table 2). The numbers were slightly different for men.

The patients did not show higher likelihood of having consulted any of the health care professionals during the period 2-12 months prior to diagnosis than the comparison subjects, except for neurology consultations. Male patients had higher odds of having 1 or 2 consultations with a neurologist, and female patients had higher odds of having 3 or more consultations with a neurologist relative to their comparison subjects (Table 2).

In general, patients with malignant tumors had significantly more consultations than the comparison subjects 1-7 months prior to diagnosis, peaking in the last month prior to diagnosis (Figures 2, 4, and S2). The starting point of increased health care use differed between male and female patients, eg, general practice (women: 4 months, men: 6 months) and neurology (women: 7 months, men: 5 months) and across medical specialties (Figures 2, 4, and S2).
The peak in consultations 2 months prior to diagnosis ranged from $61 \%$ more consultations with a GP $\left(\mathrm{IRR}_{\text {women }}=1.61\right.$ [95\% CI: $\left.\left.1.45 ; 1.79\right]\right)$ to more than 16 times more neurology consultations ( $\mathrm{IRR}_{\text {women }}=16.74[95 \% \mathrm{CI}$ : 11.32; 24.75]) (Figures 2 and 4).

\section{Subanalyses}

Overall, similar results were seen when adjusting for known specialty-specific disease (Table S1), although the ORs of having 1-2 ophthalmology consultations for women with benign tumors and of having 1-2 neurology consultations for men with benign tumors were no longer significant.

\section{Discussion \\ Main findings}

This study on data from almost 6,000 patients diagnosed with a primary intracranial tumor shows that patients diagnosed with benign and malignant primary intracranial tumors use health care differently in the time prior to diagnosis. Patients 

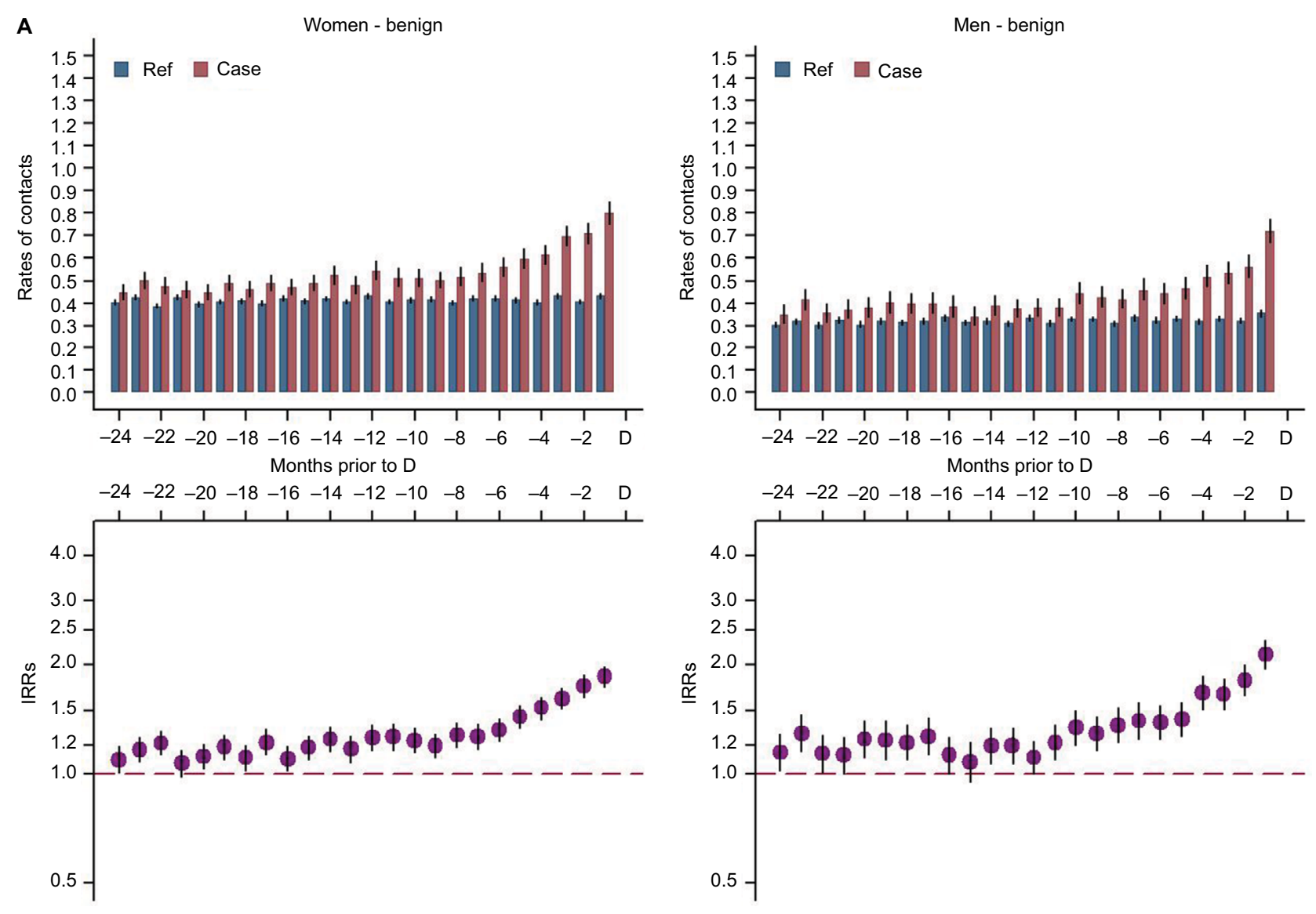

B

Women - benign
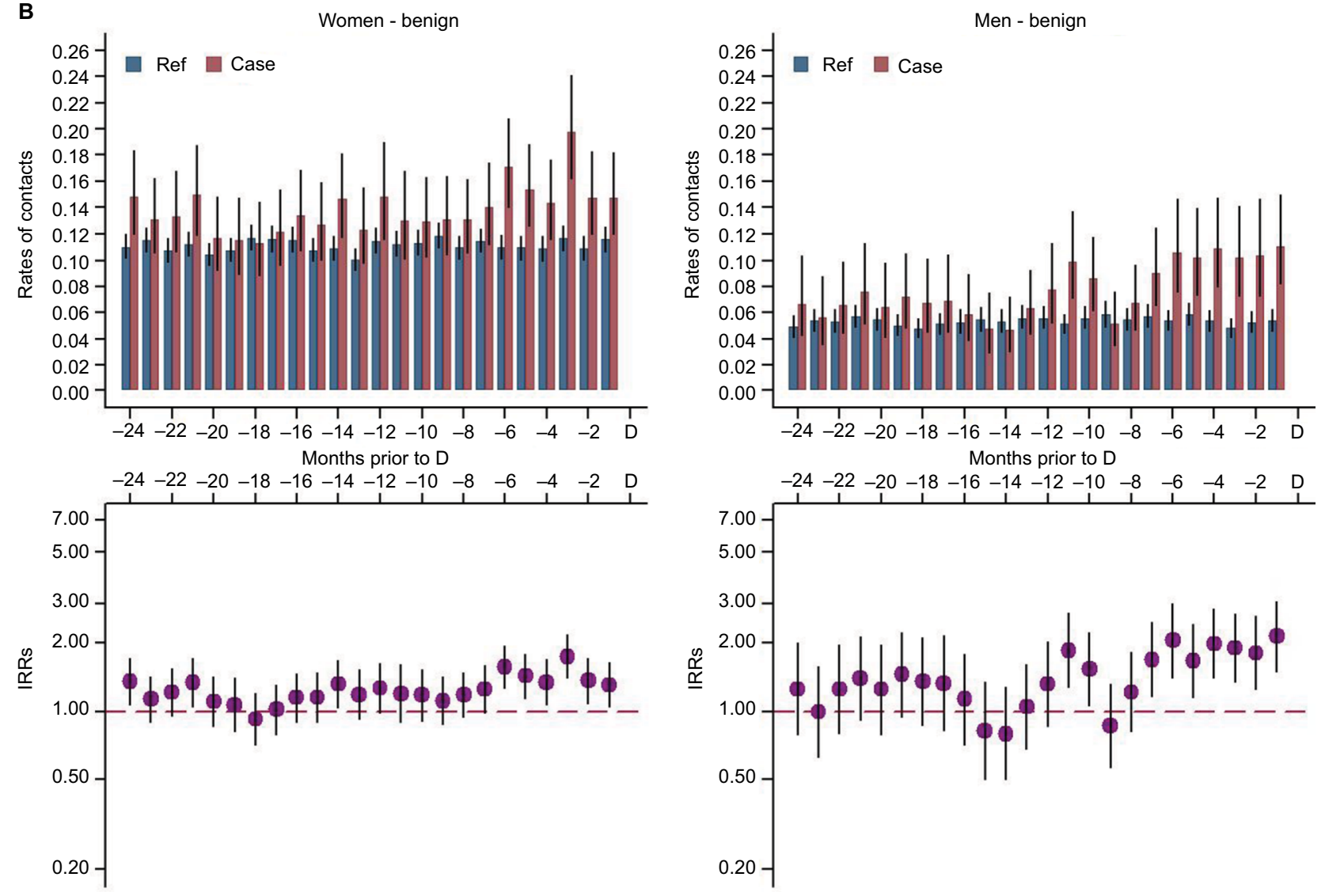

Figure I (Continued) 

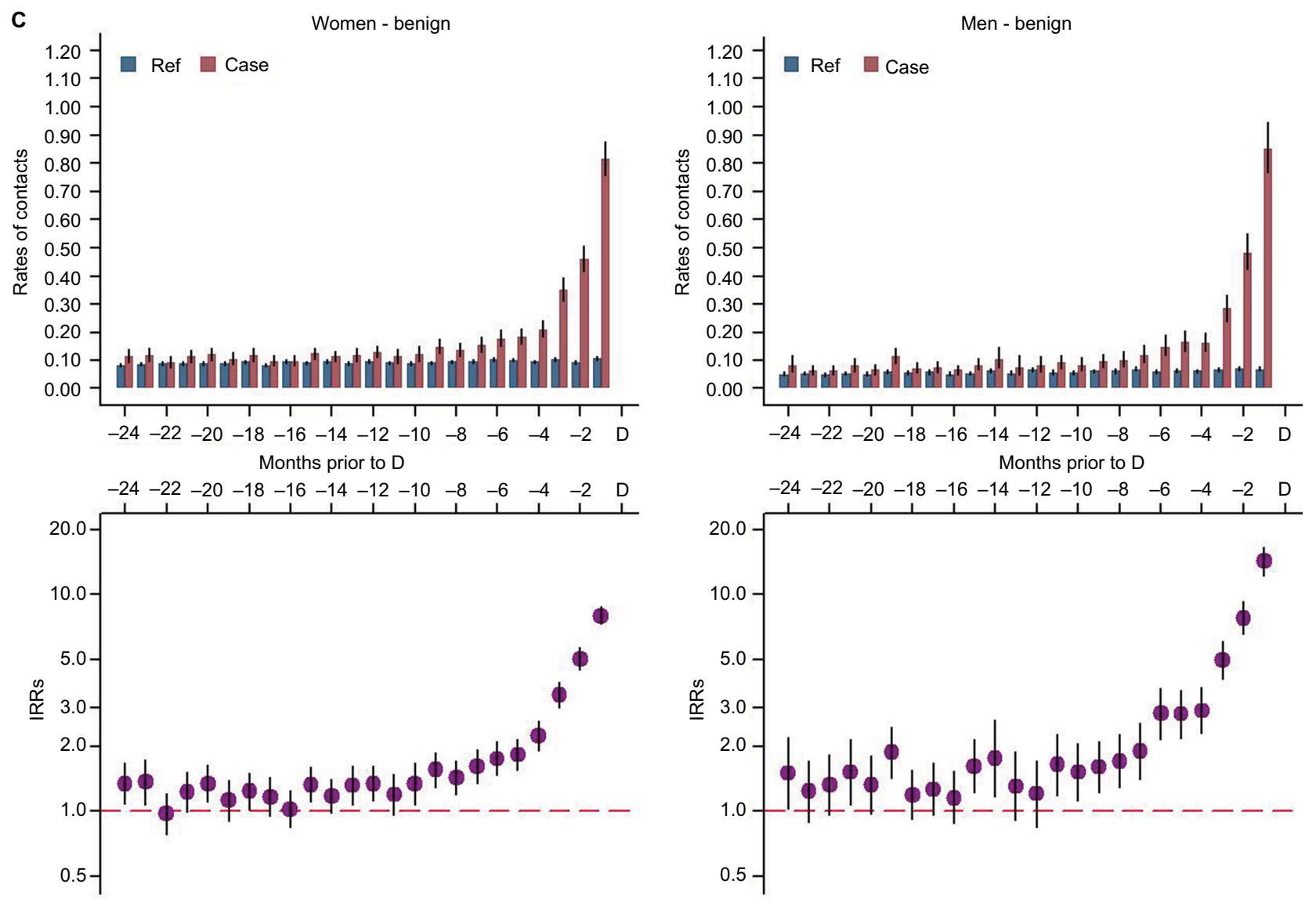

Figure I Total health care contacts to GP (daytime) (A) physiotherapy (B), and radiological examinations (C) among patients with benign primary intracranial tumors. Notes: Upper parts of A-C: average rates of contacts for patients and comparison subjects 24 months prior to diagnosis/index date. Lower parts of A-C: IRRs with $95 \%$ Cls. Abbreviations: $\mathrm{Cl}$, confidence interval; D, date of diagnosis/index date; GP, general practitioner; IRR, incidence rate ratio; Ref, reference.

with malignant tumors had increased health care use in relatively close proximity to the diagnosis, whereas patients with benign tumors had increased health care use for a long time before diagnosis. Most notably, patients with benign tumors had increased health care use 24 months prior to diagnosis for ENT consultations, from 21 months prior to diagnosis for neurology consultations, and from 12 months prior to diagnosis for ophthalmology consultations.

\section{Strengths and limitations}

The strengths of this study include the population-based design, which was permitted by the uniformly organized health care system in Denmark, the use of the data from the complete Danish registries, ${ }^{18,19,28}$ and the identification of patients in the Danish Cancer Registry. ${ }^{16}$ The comparison subjects were randomly sampled by a density sampling procedure and thus should be representative of the source population concerning health care use.
Although we used the Danish Cancer Registry to identify patients, misclassification of tumors as either malignant or benign may still occur as the diagnosis may have been based solely on neuroimaging. ${ }^{29,30}$ However, such misclassification should result in an underestimation of the associations found. Some information on educational level and labor market affiliation was missing. The missing data was distributed equally between patients and comparison subjects, and inclusion of these data should not bias the results.

We minimized the risk of confounding by matching comparison subjects and patients according to gender, age, and general practice and by adjusting the analyses for socioeconomic and demographic factors. However, residual confounding may still prevail. Yet, the risk of residual confounding should be equally distributed for both patients and comparison subjects, which is likely to lead to an underestimation of the associations.

We restricted the primary intracranial tumor population to first-time cancers only to avoid the influence of potentially 
A
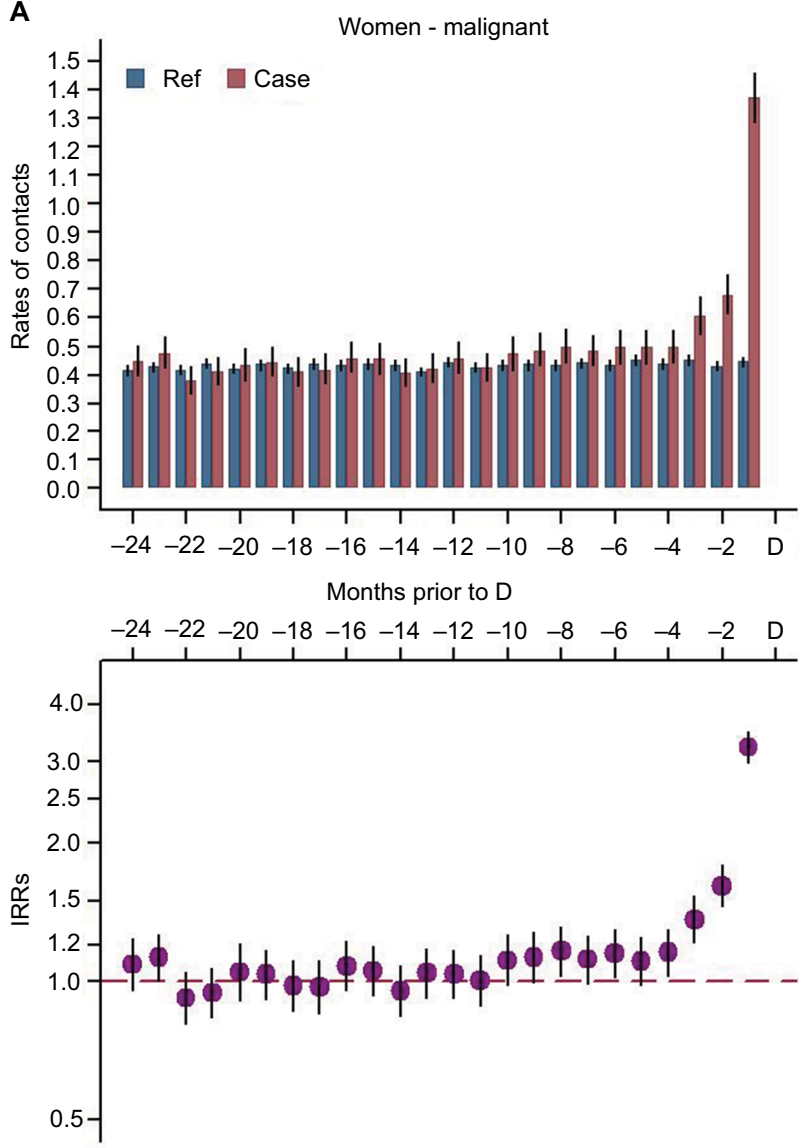

B
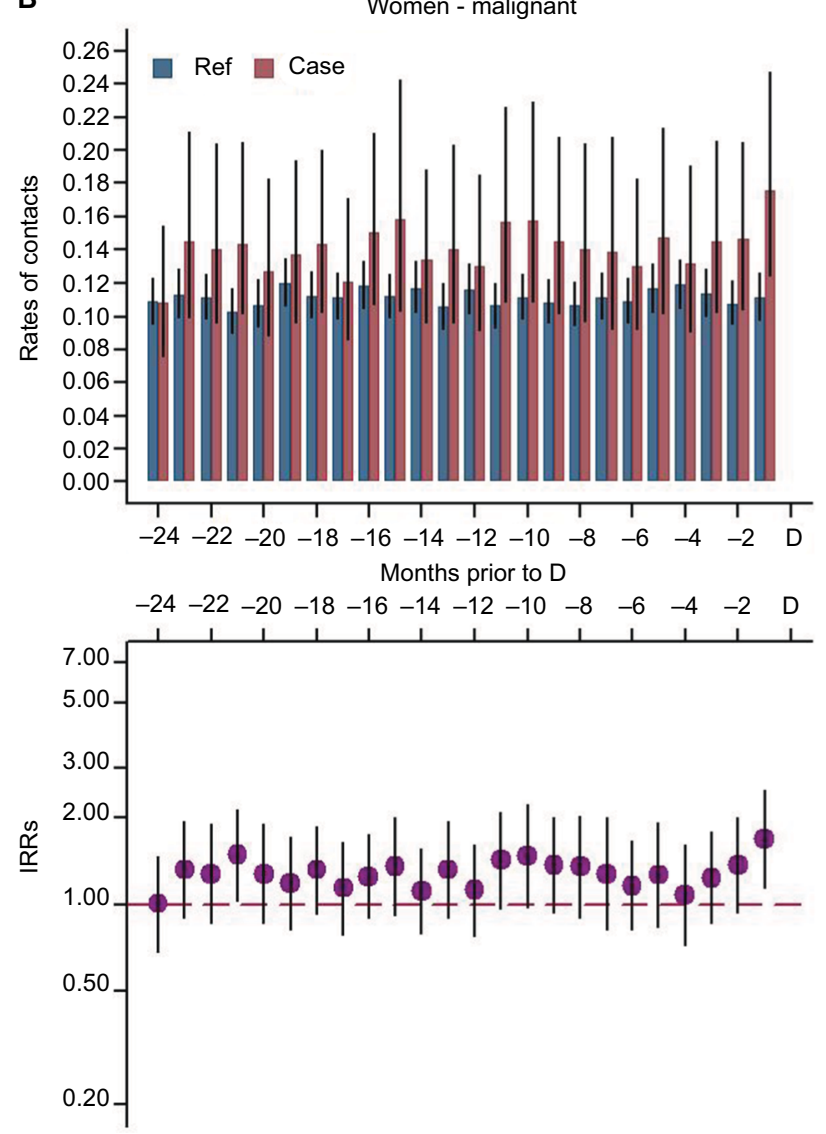

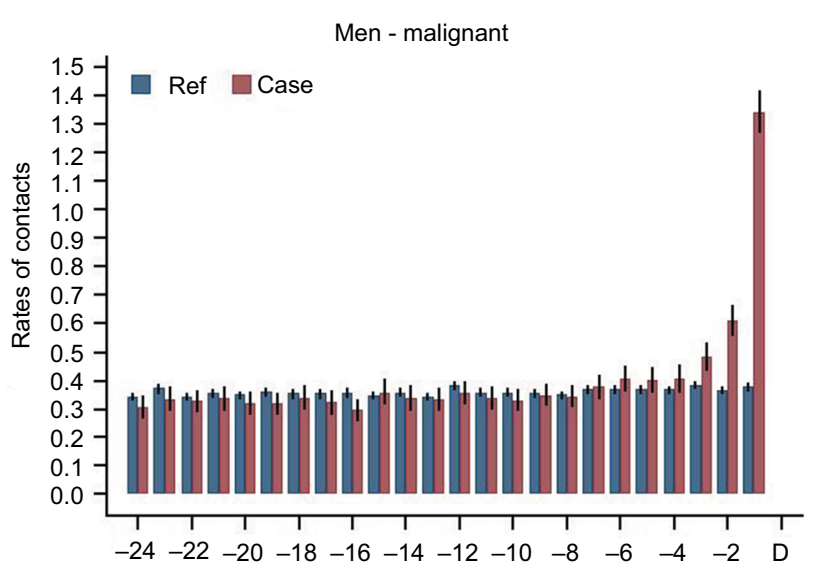

Months prior to $\mathrm{D}$

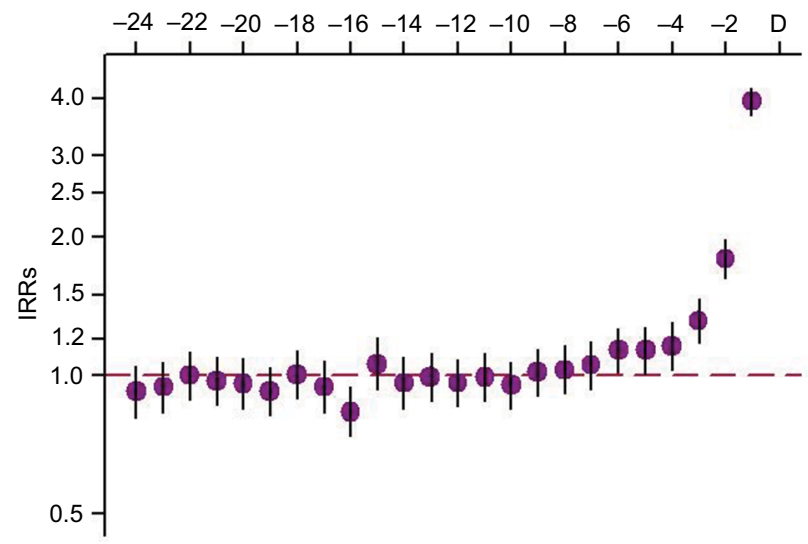

Men - malignant

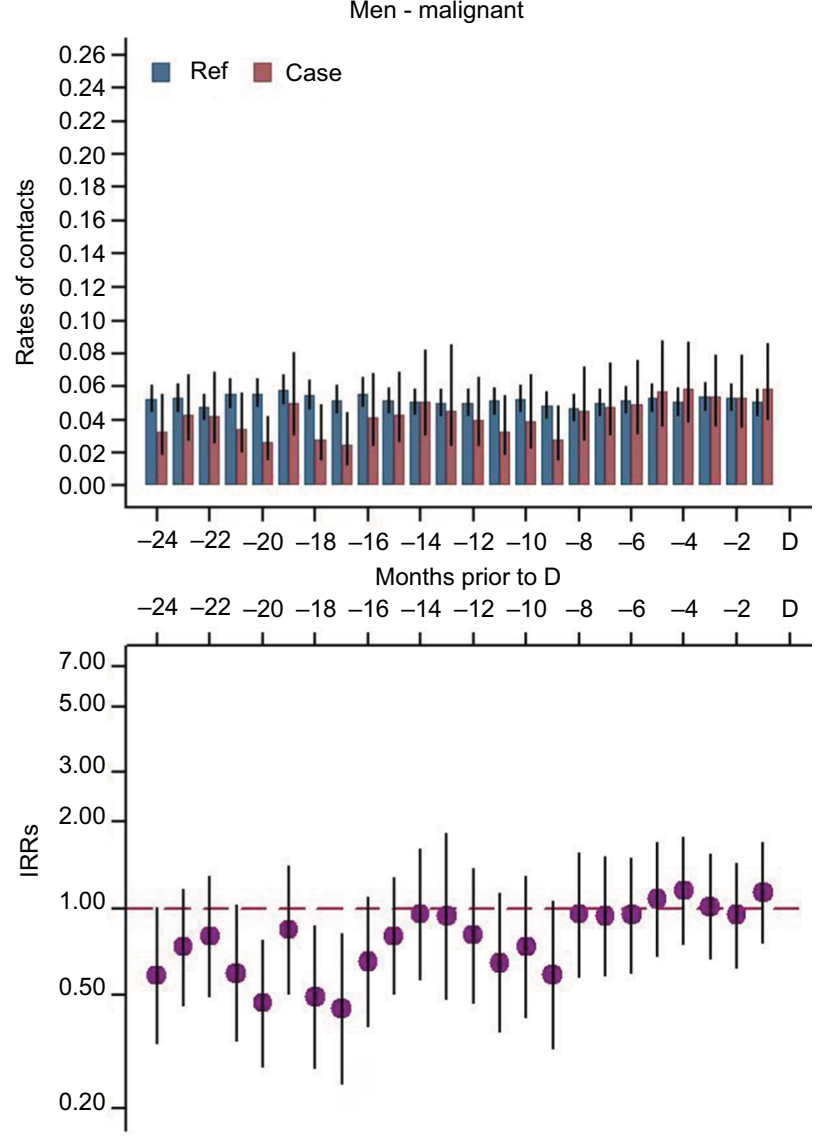

Figure 2 (Continued) 
C
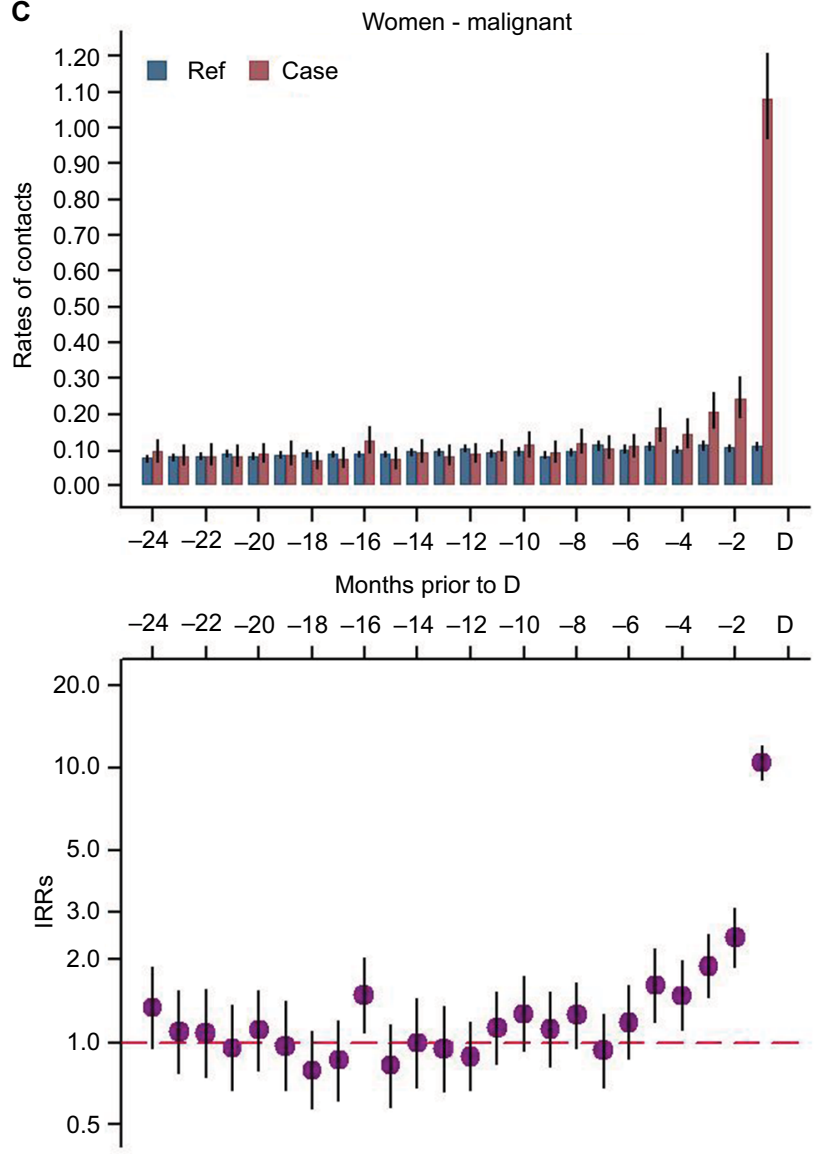
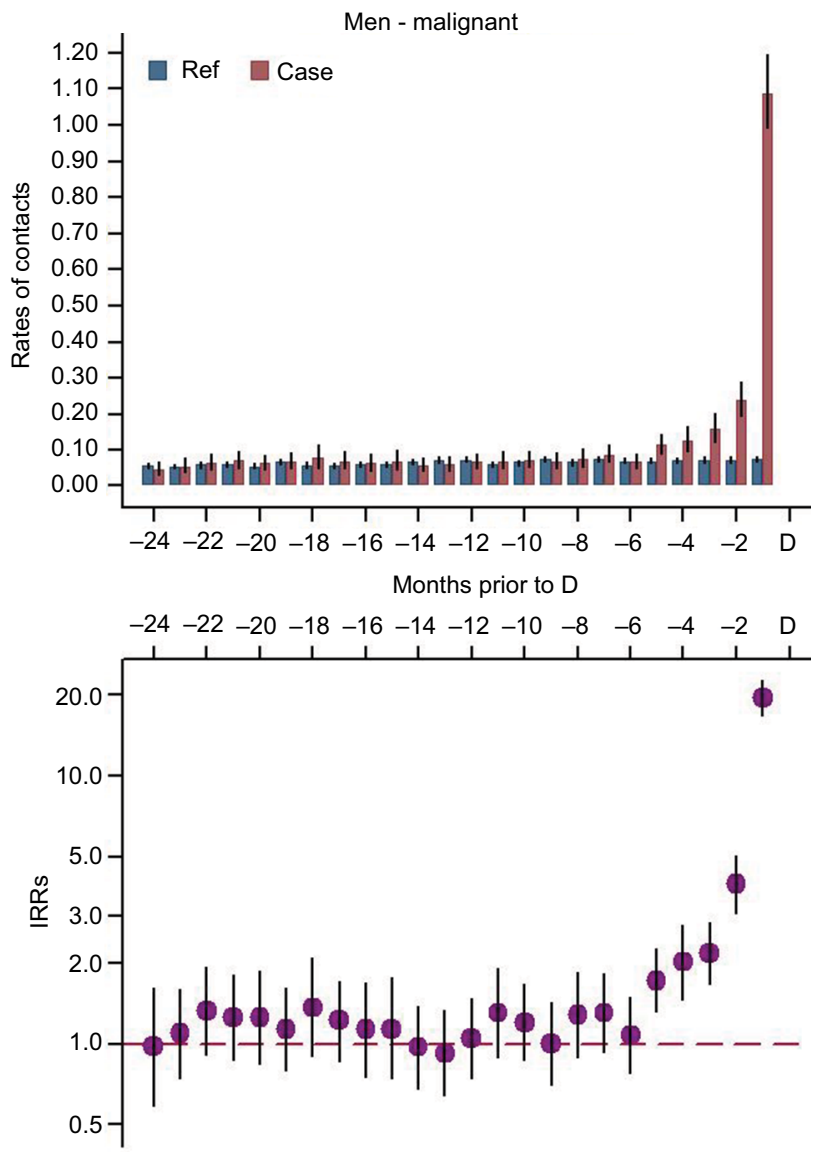

Figure 2 Total health care contacts to GP (daytime) (A) physiotherapy (B), and radiological examinations (C) among patients with malignant primary intracranial tumors. Notes: Upper parts of A-C: average rates of contacts for patients and comparison subjects 24 months prior to diagnosis/index date. Lower parts of A-C: IRRs with $95 \%$ Cls. Abbreviations: $\mathrm{Cl}$, confidence interval; D, date of diagnosis/index date; GP, general practitioner; IRR, incidence rate ratio; Ref, reference.

increased alertness of health care professionals among patients with a previous history of cancer. This decreased the risk of confounding, but it may also have decreased the generalizability of the study. Surveillance bias may play a role as the patients had more known diseases requiring consultations with medical specialists than the comparison subjects (Table $\mathrm{S} 2$ ). Yet, the results only changed slightly after adjustment for known specialty-specific diseases (Tables 2 and S1).

Although the gender-specific analyses and the benign/ malignant stratification procedure were used to acknowledge and limit the risk of confounding and selection bias, these analyses and procedures also reduced the statistical precision of the study.

For some of the outcomes, it may be argued that the individual risk of having an increased health care utilization is low, as the absolute difference in proportion is small despite a large relative difference. However, the population-based estimates still indicate possible aspects of clinical practice, which may be optimized at the individual patient level.

\section{Comparison with other studies}

We could not compare our findings directly with other studies of health care use prior to a primary intracranial tumor diagnosis in adults due to the absence of such studies. However, studies in children and young adults with CNS tumors have found increased health care use from 6 and 17 months before diagnosis, respectively. ${ }^{31,11}$ Yet, neither of these studies accounted for differences between benign and malignant tumors. Even so, our findings of increased health care use for patients with benign primary intracranial tumors for up to 2 years before diagnosis indicate that the possibility for an earlier diagnosis in adult patients with benign primary intracranial tumors may be larger than the window of opportunity found in children and young adults with CNS tumors. 
A
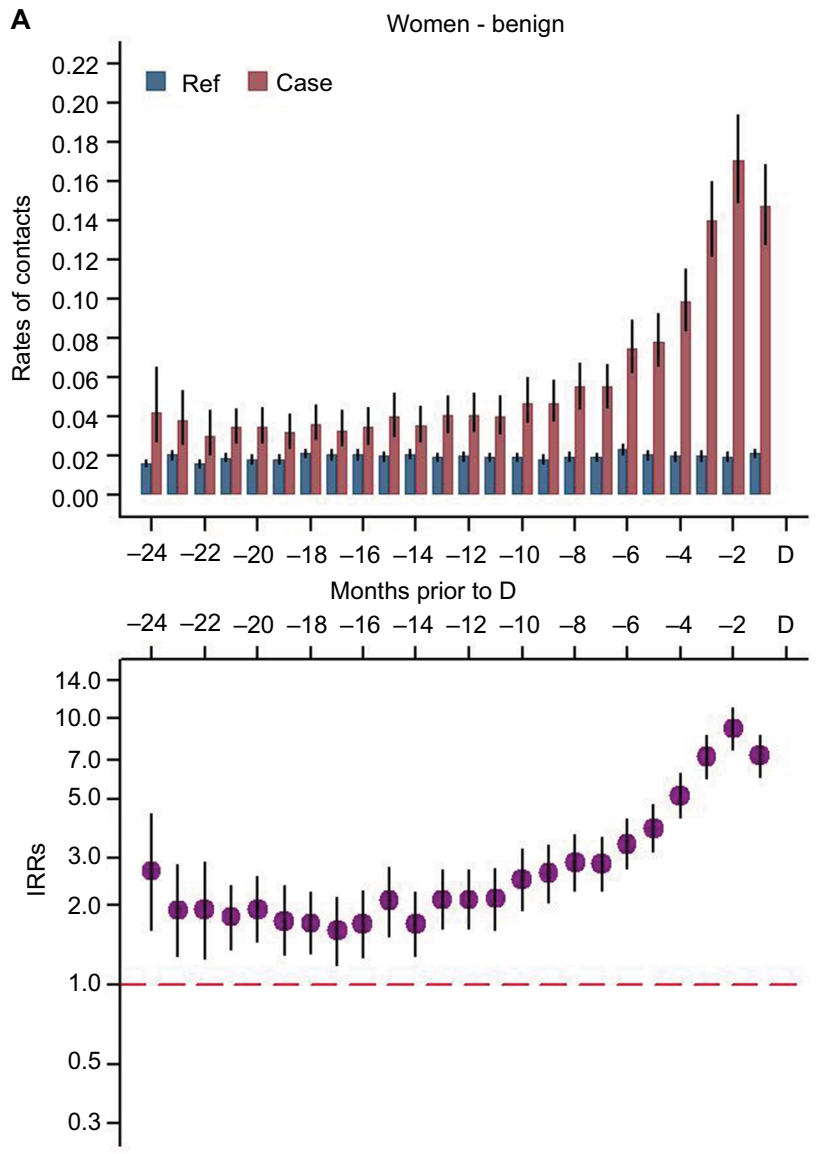

B

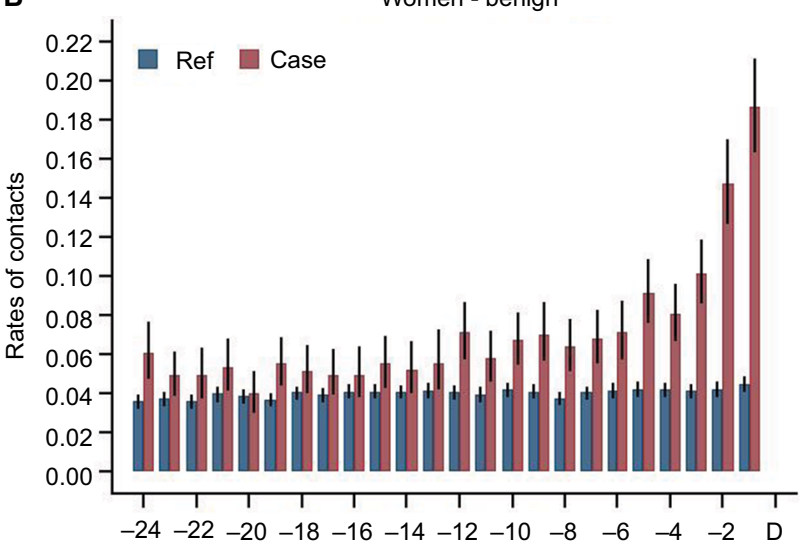

Months prior to $\mathrm{D}$

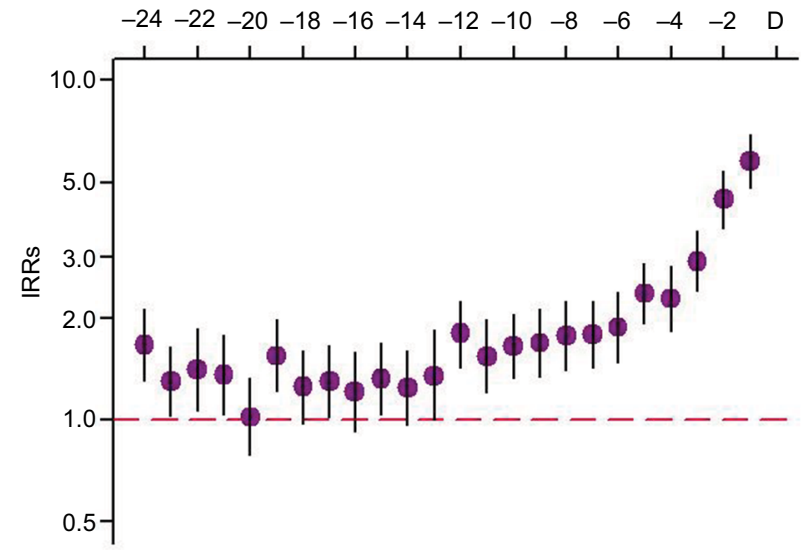

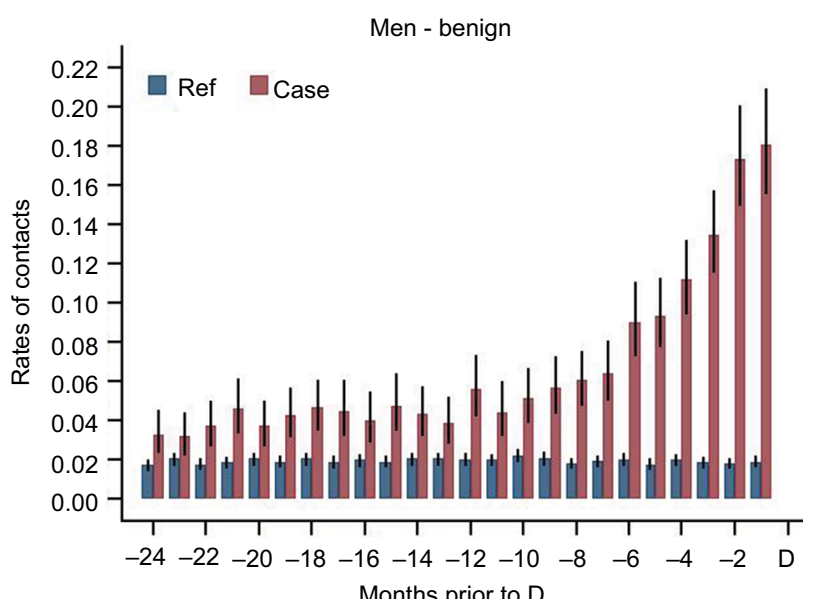
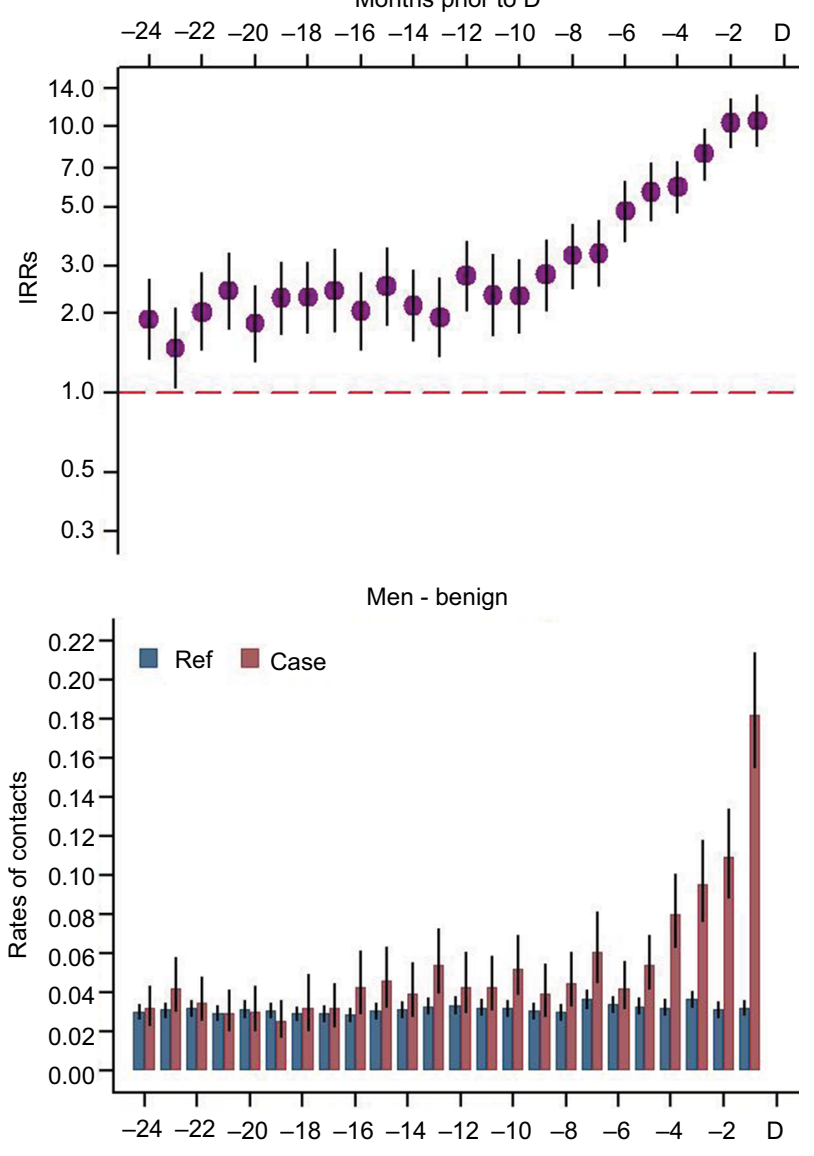

Months prior to $D$

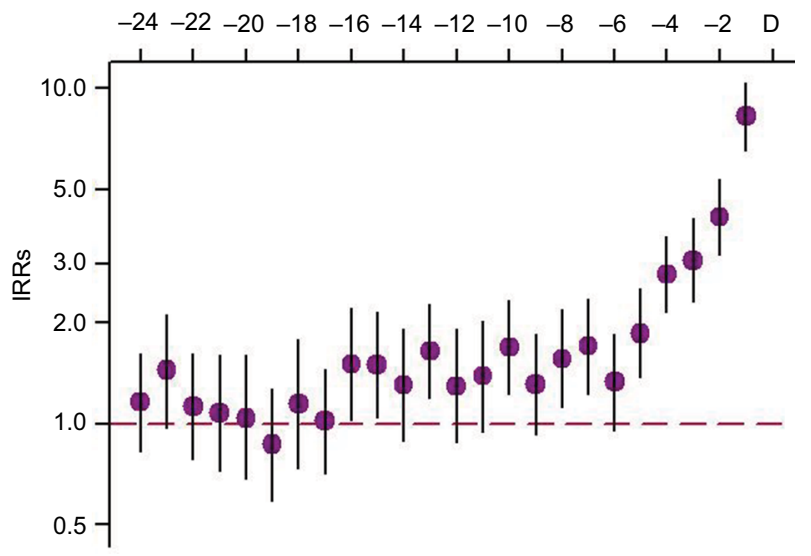

Figure 3 (Continued) 
C

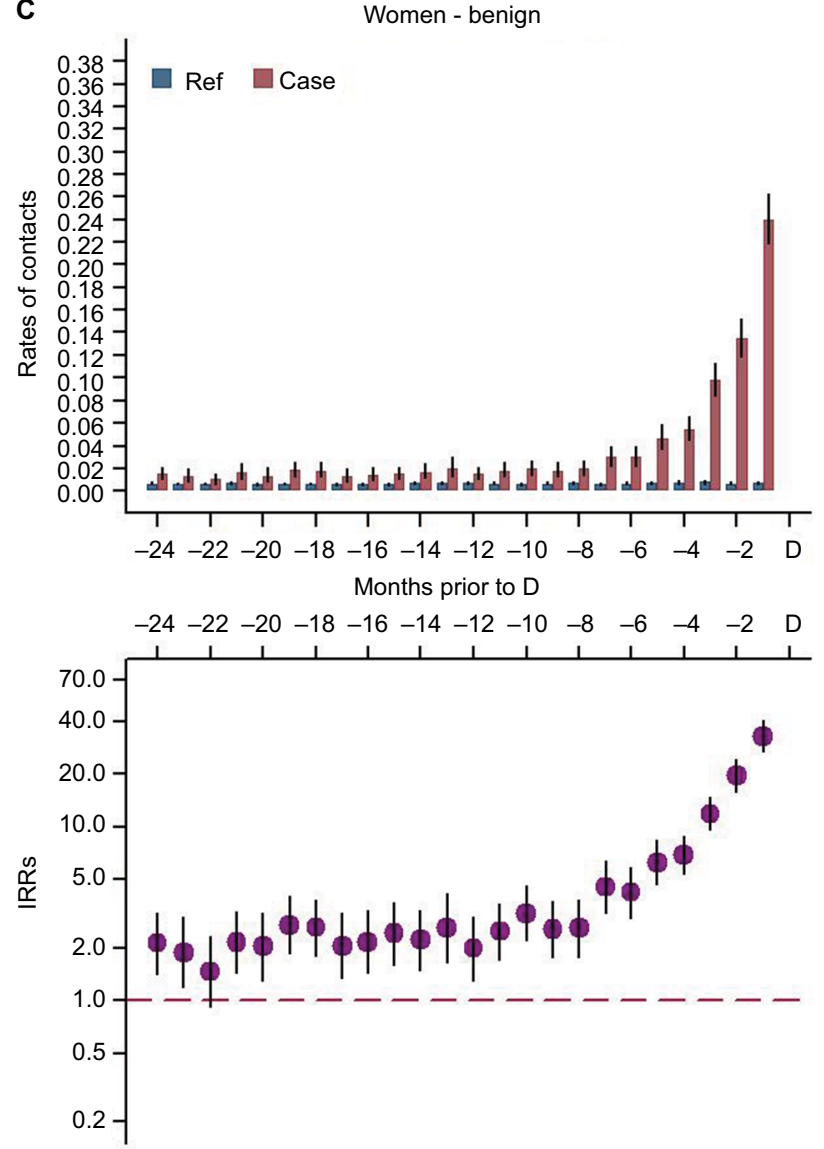

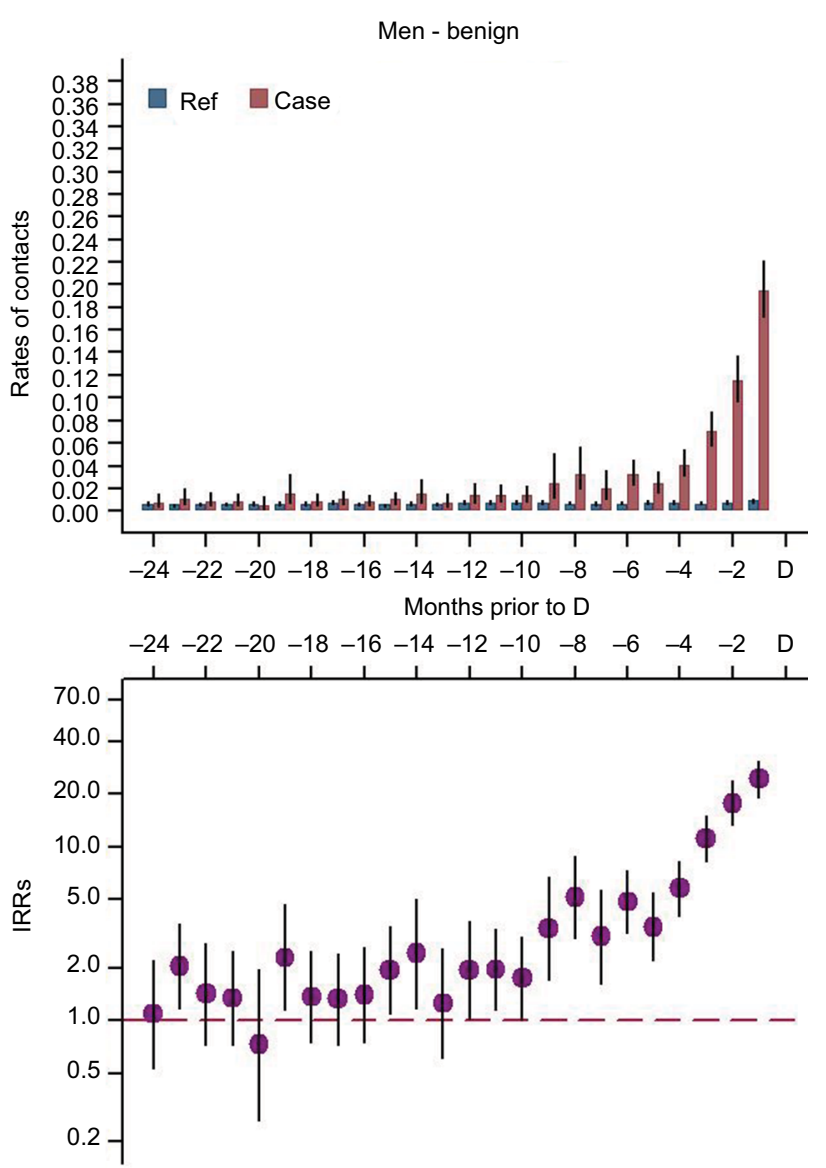

Figure 3 Total health care contacts to ENT (A), ophthalmology (B), and neurology (C) among patients with benign primary intracranial tumors.

Notes: Upper parts of A-C: average rates of contacts for patients and comparison subjects 24 months prior to diagnosis/index date. Lower parts of A-C: IRRs with $95 \%$ Cls. Abbreviations: $\mathrm{Cl}$, confidence interval; D, date of diagnosis/index date; ENT, ear-nose-throat; IRR, incidence rate ratio; Ref, reference.

Studies of other cancer types have reported increased health care use before a cancer diagnosis with a steep rise from 3 to 6 months prior to diagnosis. ${ }^{32-34}$ Interestingly, 1 study found an increased health care use up to 2 years prior to diagnosis among lung cancer patients with known chronic obstructive pulmonary disease. ${ }^{35}$ In contrast to these studies, we found that patients with malignant primary intracranial tumors only had an increase in health care use relatively close to the time of diagnosis, which indicates lower opportunity for an earlier diagnosis in this group of patients. Studies from the UK have shown that brain tumor patients are more likely to have multiple primary care consultations before referral to hospital ${ }^{12}$ and to have long primary care intervals. ${ }^{12,36}$ This may indicate that the symptomatology in patients with a primary intracranial tumor is more nonspecific and vague than in patients with other types of cancers.

In contrast to a Danish study, ${ }^{37}$ the primary intracranial tumor patients in our study had almost the same amount of psychiatric contacts than the comparison subjects. Female patients did, however, have significantly increased health care use from 4 months prior to diagnosis for benign tumors and from 2 months prior to diagnosis for malignant tumors; this is in accordance with the findings by Benros et al. ${ }^{37}$

It is well established that many patients with intracranial tumors present with first-time seizures or sudden onset of focal neurological symptoms. ${ }^{7,38-41}$ Such emergency presentations could be a marker of diagnostic delay, but it could also simply be related to the nature of intracranial tumors. ${ }^{42-44}$ Yet, it is ambiguous if these patients have had an initial symptomatic presentation in general practice that did not lead to suspicion of cancer. ${ }^{37,41}$

As many intracranial tumor patients present as emergencies and as most of the symptoms of intracranial tumors have low predictive values in general practice, ${ }^{8,45,46}$ only about $1 \%$ of adult intracranial tumors are diagnosed following a fast-track GP referral for suspected cancer in the UK..$^{40}$ In Denmark, the number of patients detected through the Danish 
A
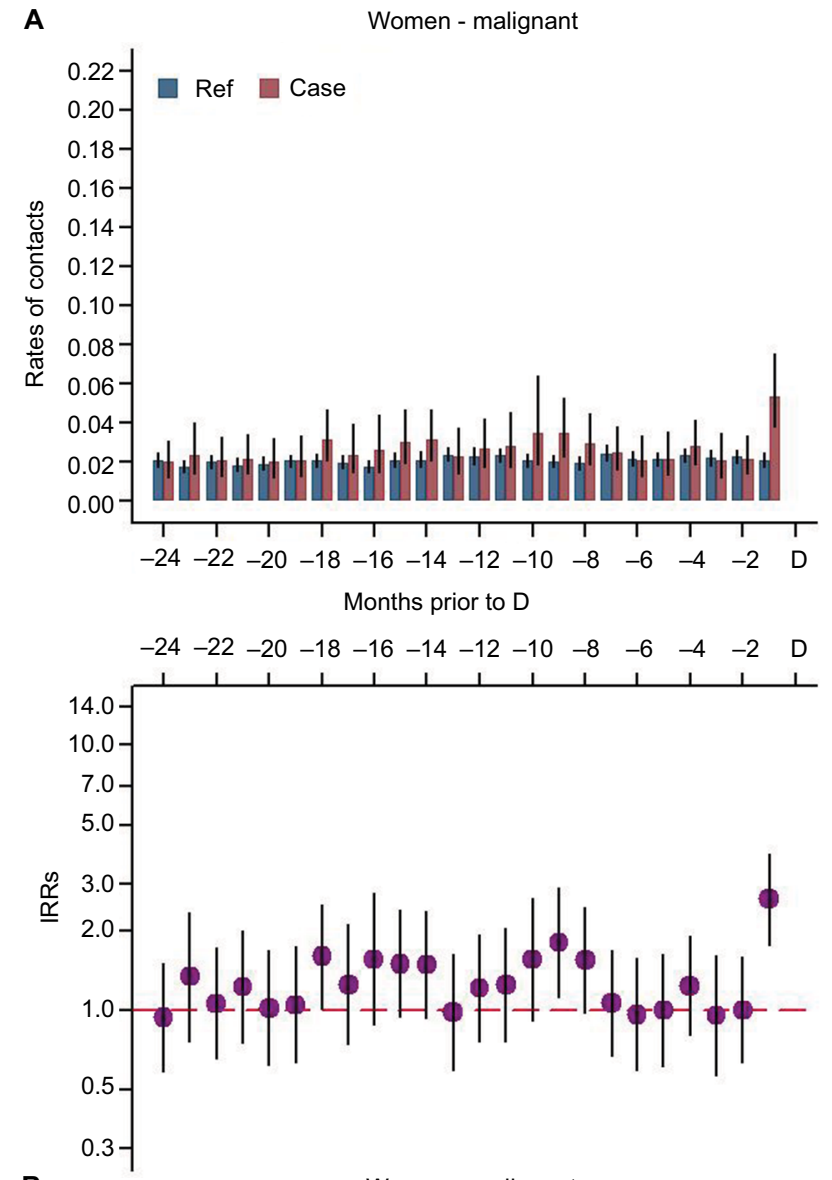

B
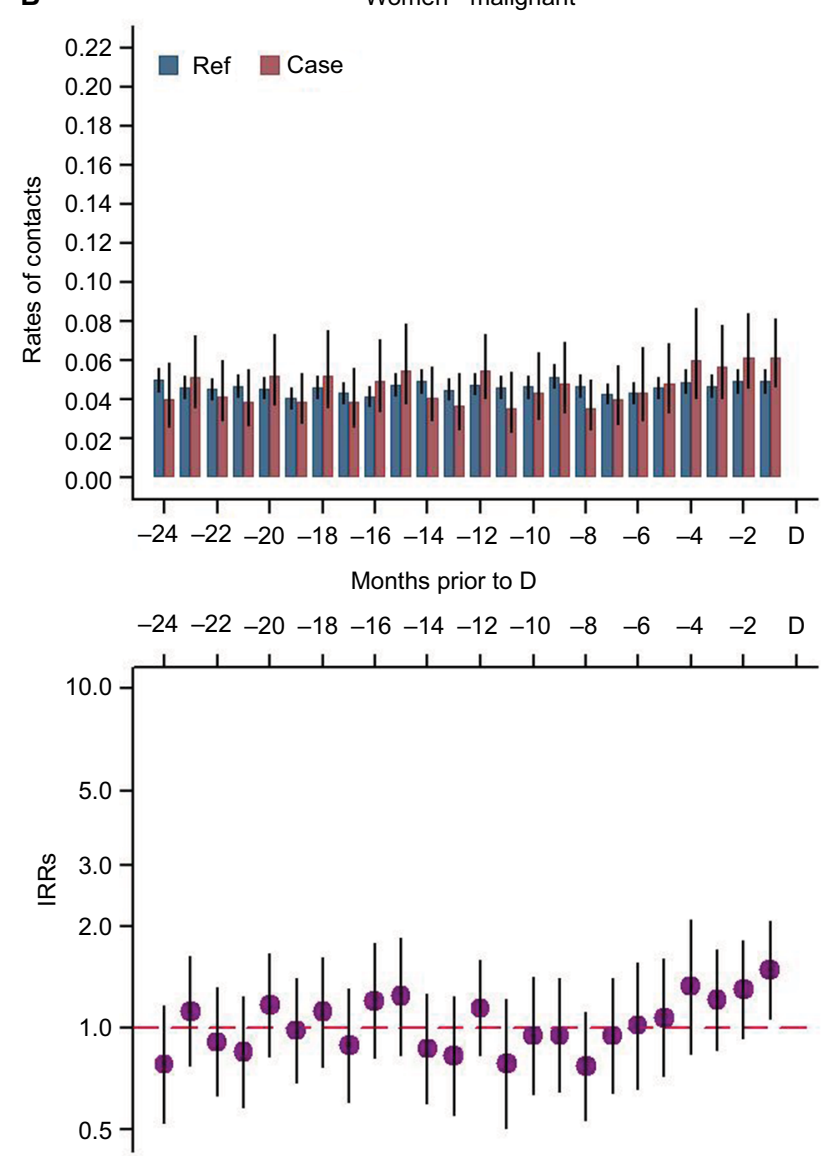
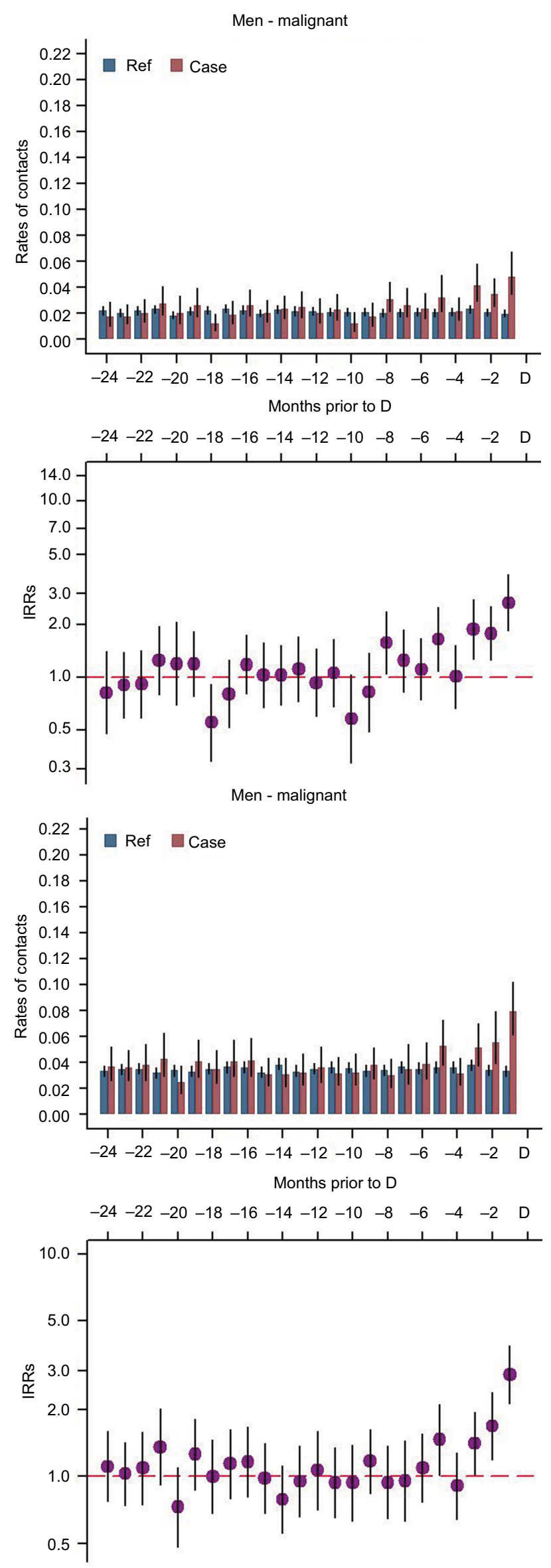

Figure 4 (Continued) 
C
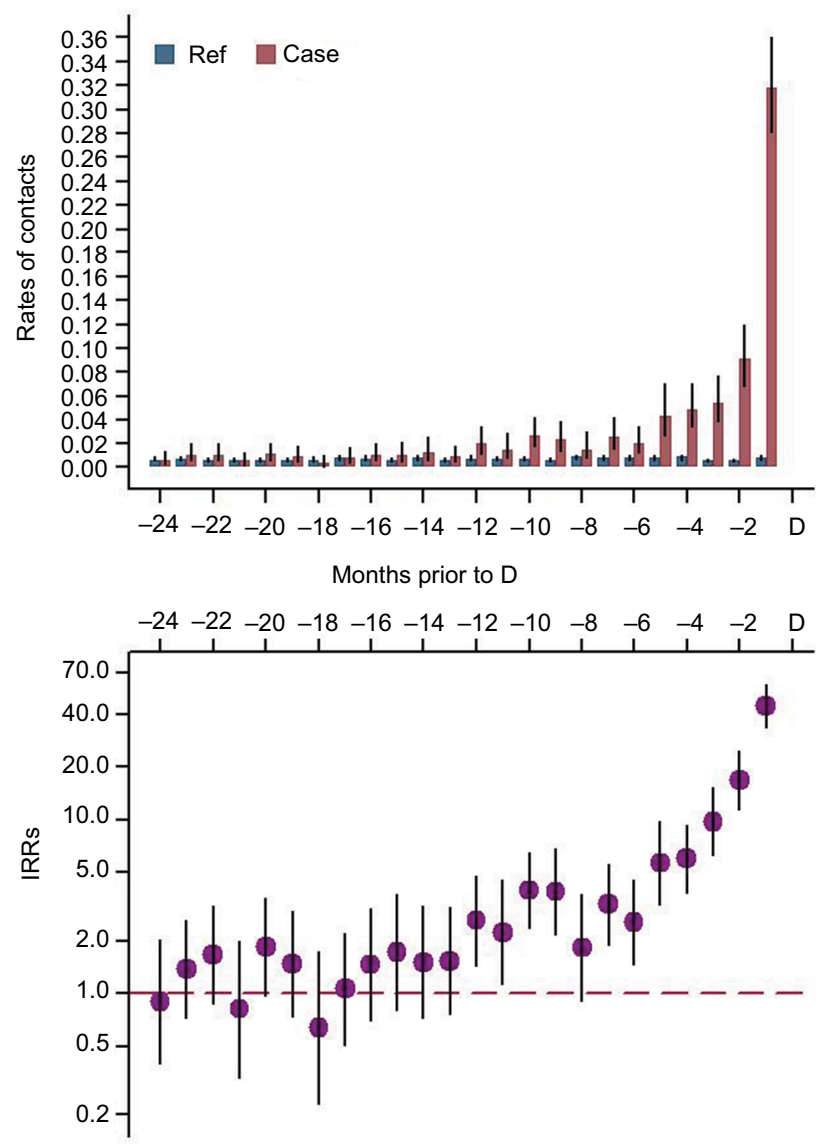

Men - malignant

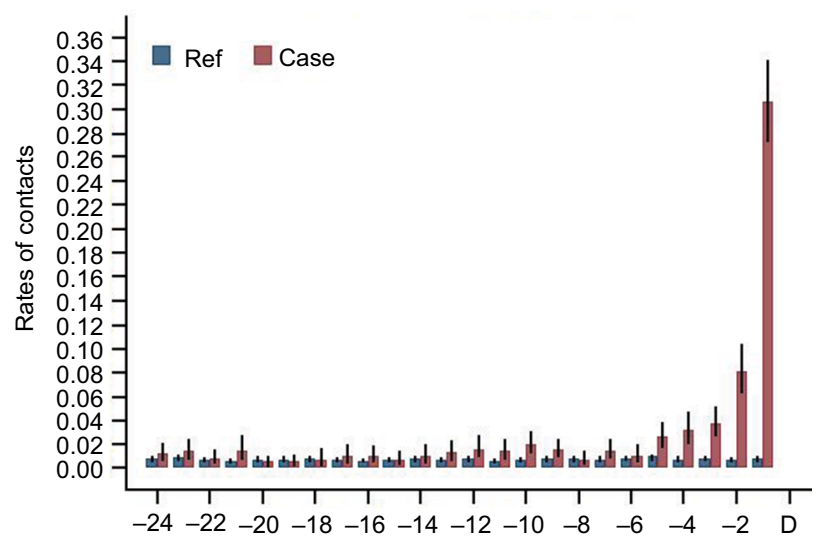

Months prior to $D$

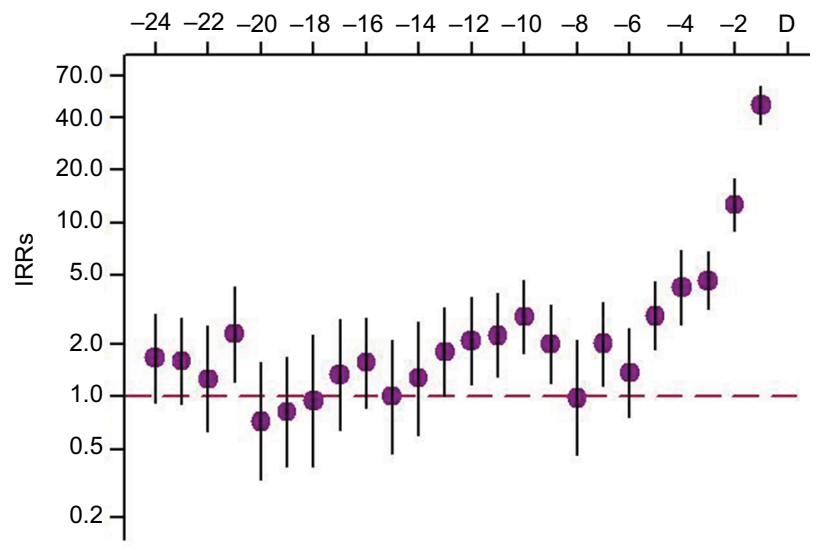

Figure 4 Total health care contacts to ENT (A), ophthalmology (B), and neurology (C) among patients with malignant primary intracranial tumors.

Notes: Upper parts of A-C: average rates of contacts for patients and comparison subjects 24 months prior to diagnosis/index date. Lower parts of A-C: IRRs with $95 \%$ Cls. Abbreviations: $\mathrm{Cl}$, confidence interval; D, date of diagnosis/index date; ENT, ear-nose-throat; IRR, incidence rate ratio; Ref, reference.

brain Cancer Patient Pathway is unknown, but more than one-third of the patients diagnosed with an intracranial tumor were initially referred on suspicion of other neurological illnesses. ${ }^{45}$ Although the Danish brain Cancer Patient Pathway has been found to have acceptable diagnostic work-up time, our study indicates that the diagnosis may be achievable much sooner, as especially patients with a benign tumor have increased health care activity involving several medical specialists many months before diagnosis.

\section{Conclusion}

Patients diagnosed with benign and malignant primary intracranial tumors use health care services differently. The increased health care use seen among patients with benign primary intracranial tumors several months prior to diagnosis indicates that there may be a window of opportunity to diagnose these patients several months earlier. In contrast, for patients with malignant primary intracranial tumors, the increased health care use occurred within relatively close proximity to the diagnosis, which indicates lower opportunity for earlier diagnosis in this group of patients.

Future studies should focus on the reasons behind the increased health care use before diagnosis for the patients diagnosed with a benign primary intracranial tumor in order to optimize the diagnostic process.

\section{Acknowledgments}

The authors wish to thank statistician Anders Helles Carlsen for assistance with the statistical analyses, Kaare Rud Flarup for his assistance with data retrieval from the national registries, and Lone Niedziella for assistance regarding scientific writing in English. The study was funded by an unrestricted grant from the Lundbeck Foundation (grant number R1972015-4116). Data infrastructure and supervision was funded by the Research Centre for Cancer Diagnosis in Primary Care $(\mathrm{CaP})$ through the Danish Cancer Society. None of the 
funding sources had a role in the design, conduct, analysis, or reporting of the study.

\section{Disclosure}

The authors report no conflicts of interests in this work.

\section{References}

1. Freitag MT, Maier-Hein KH, Binczyk F, et al. Early detection of malignant transformation in resected WHO II low-grade glioma using diffusion tensor-derived quantitative measures. PLoS One. 2016;11(10):1-19.

2. Schafalitzky De Muckadell O, editor. Medicinsk Kompendium [Compendium in Medicine]. Vol 2. 18th ed. Copenhagen: Nyt Nordisk Forlag Arnold Busck; 2013.

3. Jensen H, Tørring ML, Larsen MB, Vedsted P. Existing data sources for clinical epidemiology: Danish cancer in primary care cohort. Clin Epidemiol. 2014;6:237-246.

4. Hansen AH, Halvorsen PA, Ringberg U, Førde OH. Socio-economic inequalities in health care utilisation in Norway: a population based cross-sectional survey. BMC Health Serv Res. 2012;12:336.

5. Sundhedsstyrelsen. Pakkeforløb for kræft i Hjernen 2013 [the Danish brain Cancer Patient Pathway 2013]. Copenhagen, Denmark: Sundhedsstyrelsen. Available from: https://www.sst.dk/da/sygdom-ogbehandling/kraeft/pakkeforloeb/ /media/7FD13233FEA2447B95D22 469AEEDCD72.ashx. Accessed March 22, 2017.

6. Nielsen TN, Hansen RP, Vedsted P. Præsentation af symptomer i almen praksis hos patienter med cancer [Presentation of symptoms in general practice among patients with cancer]. Ugeskr Lager. 2010;172(41): 2827-2831. Danish.

7. Schmidt-Hansen M, Berendse S, Hamilton W. Symptomatic diagnosis of cancer of the brain and central nervous system in primary care: a systematic review. Fam Pract. 2015;32(6):618-623.

8. Hamilton W, Kernick D. Clinical features of primary brain tumours: a case-control study using electronic primary care records. Br J Gen Pract. 2007;57(542):695-699.

9. Rubin G, Berendsen A, Crawford SM, et al. The expanding role of primary care in cancer control. Lancet Oncol. 2015;16(12):1231-1272.

10. Walker D, Hamilton W, Walter FM, Watts C. Strategies to accelerate diagnosis of primary brain tumors at the primary - secondary care interface in children and adults. CNS Oncol. 2013;2(5):447-462.

11. Ahrensberg JM, Fenger-Grøn M, Vedsted P. Use of primary care during the year before childhood cancer diagnosis: a nationwide populationbased matched comparative study. PLoS One. 2013;8(3):1-8.

12. Lyratzopoulos G, Neal RD, Barbiere JM, Rubin GP, Abel GA. Variation in number of general practitioner consultations before hospital referral for cancer: findings from the 2010 National Cancer Patient Experience Survey in England. Lancet Oncol. 2012;13(4):353-365.

13. Pedersen C. The Danish Civil Registration System. Scand J Public Health. 2011;39(Suppl 7):22-25.

14. Mainz J, Kristensen S, Bartels P. Quality improvement and accountability in the Danish health care system. Int J Qual Health Care. 2015;27(6): 523-527.

15. Christiansen T. Organization and financing of the Danish health care system. Health Policy. 2002;59(2):107-118.

16. Gjerstorff ML. The Danish Cancer Registry. Scand J Public Health. 2011;39(Supp1 7):42-45.

17. World Health Organization. International Classification of Diseases (ICD). Geneva: World Health Organization; 2015. Available from: http:// www.who.int/classifications/icd/en/. Accessed December 13, 2016.

18. Andersen JS, Olivarius Nde F, Krasnik A. The Danish National Health Service Register. Scand J Public Health. 2011;39(Suppl 7):34-37.

19. Lynge E, Sandegaard JL, Rebolj M. The Danish National Patient Register. Scand J Public Health. 2011;39(Suppl 7):30-33.

20. Mors O, Perto GP, Mortensen PB. The Danish Psychiatric Central Research Register. Scand J Public Health. 2011;39(Suppl 7):54-57.
21. Sundararajan V, Quan H, Halfon P, et al. Cross-national comparative performance of three versions of the ICD-10 Charlson index. Med Care. 2007;45(12):1210-1215.

22. Statistics Denmark. Quality declerations. Copenhagen, Denmark: Statistics Denmark. Available from: http://www.dst.dk/en/Statistik/ dokumentation. Accessed March 21, 2017.

23. OECD. What are equivalence scales? Paris, France: OECD. 2011. Available from: http://www.oecd.org/els/soc/OECD-Note-EquivalenceScales.pdf. Accessed March 21, 2017.

24. UNESCO. The International Standard Classification of Education 2011. Comparative Social Research. Paris, France: UNESCO. 2012. Available from: http://www.uis.unesco.org/Education/Documents/isced-2011-en. pdf. Accessed April 3, 2017.

25. Statistics Denmark. Uddannelsesstatistikkens manual [Manual of Educational statistics]. Copenhagen, Denmark: Statistics Denmark. 2016.

26. Statistics Denmark. SOCIO02-Socioøkonomisk klassifikation fra 2002 [Socioeconomic classification level from 2002]. Copenhagen, Denmark: Statistics Denmark. 2002. Available from: https://www.dst. $\mathrm{dk} / \mathrm{da} /$ TilSalg/Forskningsservice/Dokumentation/hoejkvalitetsvariable/ personers-tilknytning-til-arbejdsmarkedet-set-over-hele-aaret--akm-/ socio02. Accessed March 8, 2018.

27. Juel K, Christensen K. Are men seeking medical advice too late? Contacts to general practitioners and hospital admissions in Denmark 2005. J Public Health. 2008;30(1):111-113.

28. Schmidt M, Pedersen L, Sørensen HT. The Danish Civil Registration System as a tool in epidemiology. Eur J Epidemiol. 2014;29(8): $541-549$.

29. Dansk Neuro Onkologisk Gruppe. Retningslinjer for behandling af intrakranielle gliomer hos voksne [Guidelines for treatment of intracranial gliomas in adults]. Denmark: Dansk Neuro Onkologisk Gruppe. 2016. Available from: http://www.dnog.dk/assets/files/Retningslinier PDF/ DNOG 2016 Gliom retningslinje final.pdf. Accessed March 22, 2017.

30. Young RJ, Knopp EA. Brain MRI: tumor evaluation. J Magn Reson Imaging. 2006;24(4):709-724.

31. Chu TPC, Shah A, Walker D, Coleman MP. Where are the opportunities for an earlier diagnosis of primary intracranial tumours in children and young adults? Eur J Paediatr Neurol. 2017;21(2):388-395.

32. Christensen KG, Fenger-Grøn M, Flarup KR, Vedsted P. Use of general practice, diagnostic investigations and hospital services before and after cancer diagnosis - a population-based nationwide registry study of 127,000 incident adult cancer patients. BMC Health Serv Res. 2012;12:224.

33. Hansen PL, Hjertholm P, Vedsted P. Increased diagnostic activity in general practice during the year preceding colorectal cancer diagnosis. Int J Cancer. 2015;137(3):615-624.

34. Biswas M, Ades AE, Hamilton W. Symptom lead times in lung and colorectal cancers: what are the benefits of symptom-based approaches to early diagnosis? Br J Cancer. 2015;112(2):271-277.

35. Guldbrandt LM, Møller H, Jakobsen E, Vedsted P. General practice consultations, diagnostic investigations, and prescriptions in the year preceding a lung cancer diagnosis. Cancer Med. 2017:6(1):79-88.

36. Lyratzopoulos G, Abel GA, Mcphail S, Neal RD, Rubin GP. Measures of promptness of cancer diagnosis in primary care: secondary analysis of national audit data on patients with 18 common and rarer cancers. Br J Cancer. 2013;108(3):686-690.

37. Benros ME, Laursen TM, Dalton SO, Mortensen PB. Psychiatric disorder as a first manifestation of cancer: a 10-year population-based study. Int J Cancer. 2009;124(12):2917-2922.

38. Laursen EL, Rasmussen BK. A brain cancer pathway in clinical practice. Dan Med J. 2012;59(5):A4437.

39. Lynam LM, Lyons MK, Drazkowski JF, et al. Frequency of seizures in patients with newly diagnosed brain tumors: a retrospective review. Clin Neurol Neurosurg. 2007;109(7):634-638.

40. NCIN. Routes to Diagnosis. London: National Cancer Registration and Analysis Service. Available from: http://www.ncin.org.uk/publications/ routes_to_diagnosis. Accessed March 21, 2017. 
41. Elliss-Brookes L, McPhail S, Ives A, et al. Routes to diagnosis for cancer - determining the patient journey using multiple routine data sets. Br J Cancer. 2012;107(8):1220-1226.

42. Webb AJS, Butterworth RJ. Determinants of clinical effectiveness and significant neurological diagnoses in an urgent brain cancer referral pathway in the United Kingdom. Clin Neurol Neurosurg. 2015;132:37-40.

43. Hamilton W. Emergency admissions of cancer as a marker of diagnostic delay. Br J Cancer. 2012;107(8):1205-1206.
44. Zhou Y, Abel GA, Hamilton W, et al. Defining, measuring and preventing the diagnosis of cancer as an emergency: a critical review of current evidence. Clin Oncol. 2017;14(1):45-57.

45. Laursen EL, Rasmussen BK. Work-up times in an integrated brain cancer pathway. Dan Med J. 2012;59(5):1-5.

46. Chu TPC, Shah A, Walker D, Coleman MP. Pattern of symptoms and signs of primary intracranial tumours in children and young adults: a record linkage study. Arch Dis Child. 2015;100(12):1115-1122. 


\section{Supplementary materials}

A
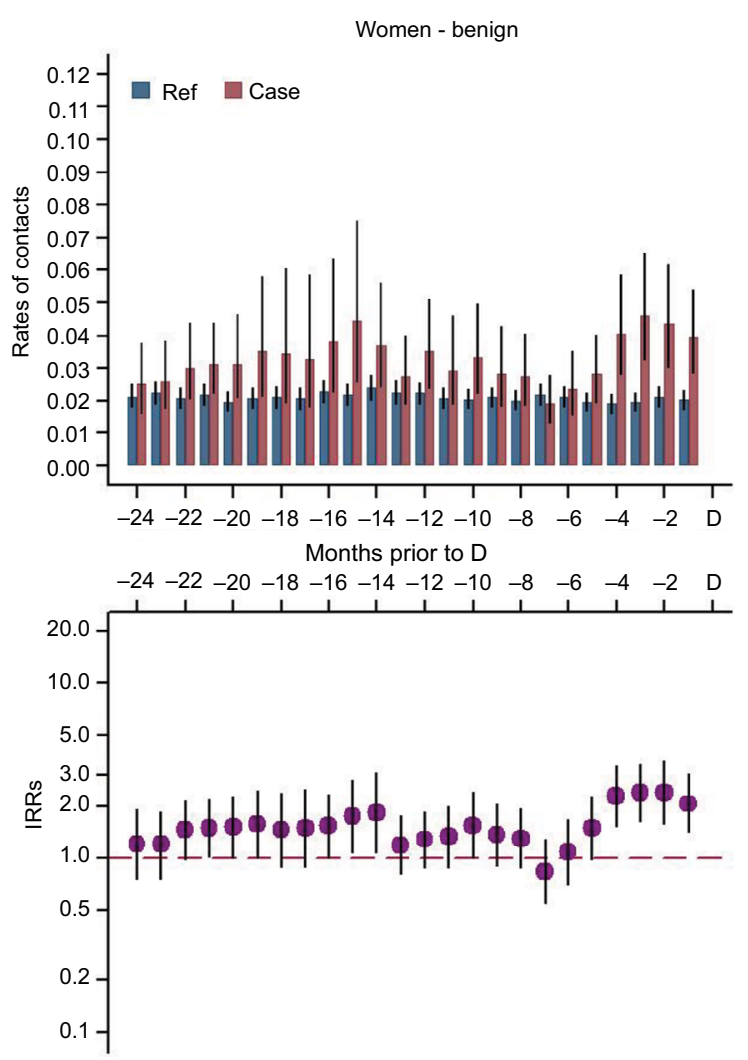

B
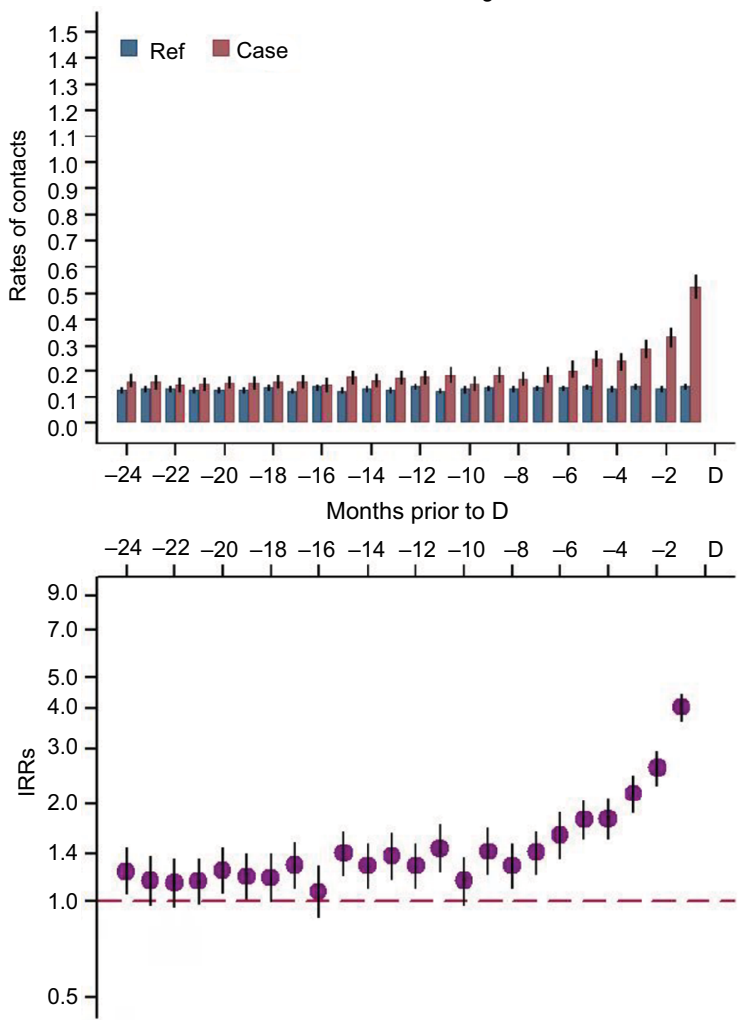
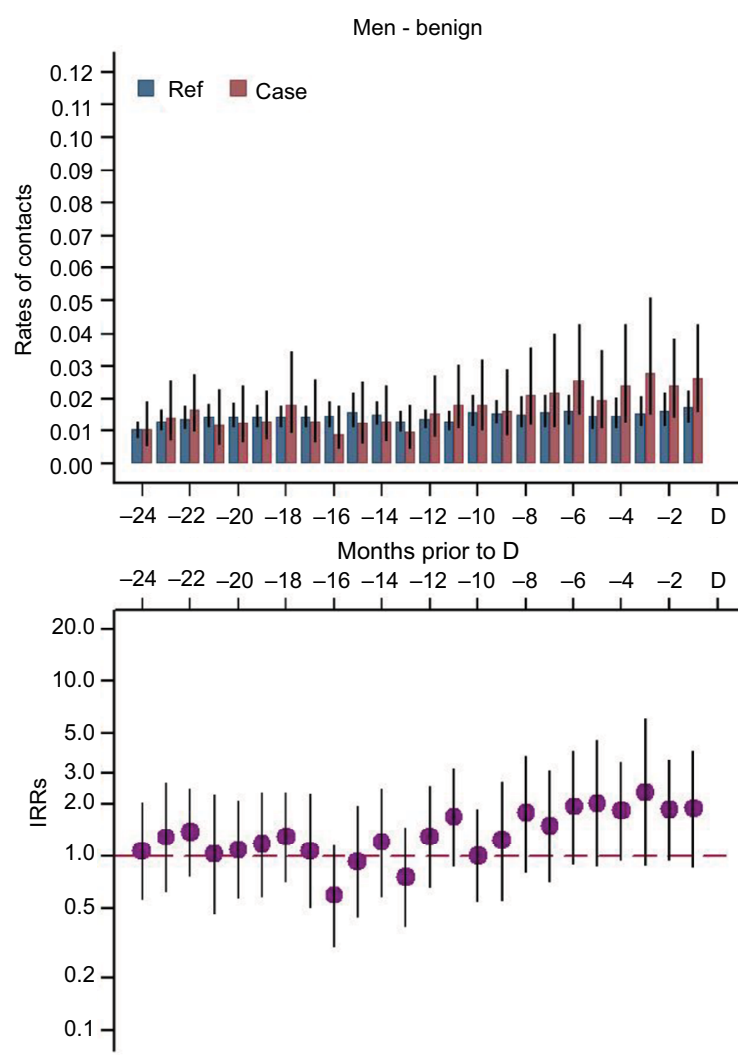

Men - benign
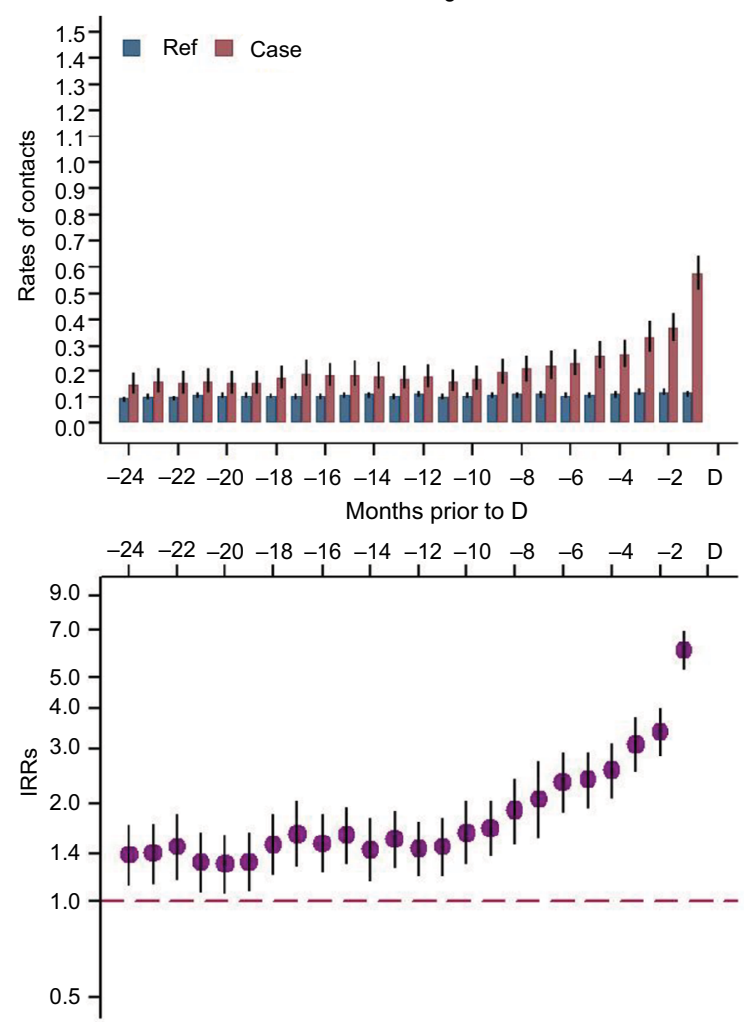

Figure SI Total health care contacts to psychiatry $(\mathbf{A})$ and other hospital contacts (B) among patients with benign primary intracranial tumors.

Notes: Upper parts of $\mathbf{A}$ and $\mathbf{B}$ : average rates of contacts for patients and comparison subjects 24 months prior to diagnosis/index date. Lower parts of $\mathbf{A}$ and $\mathbf{B}$ : IRRs with $95 \%$ Cls.

Abbreviations: $\mathrm{Cl}$, confidence interval; D, date of diagnosis/index date; IRR, incidence rate ratio; Ref, reference. 
A
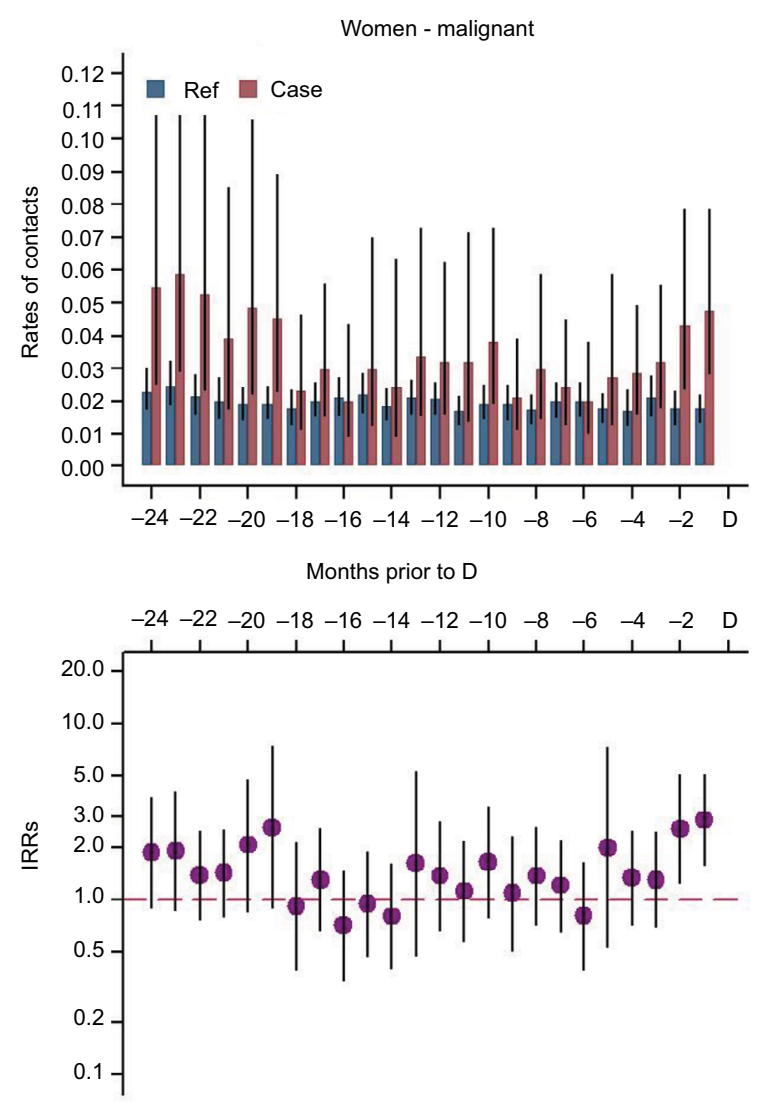

B
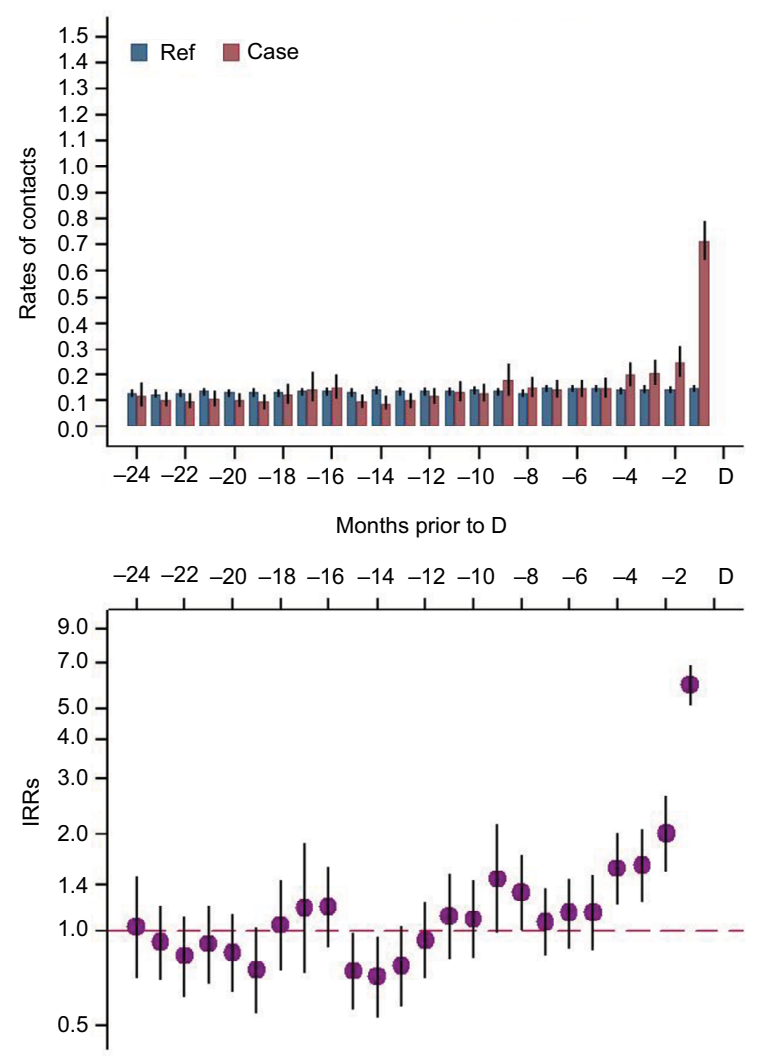
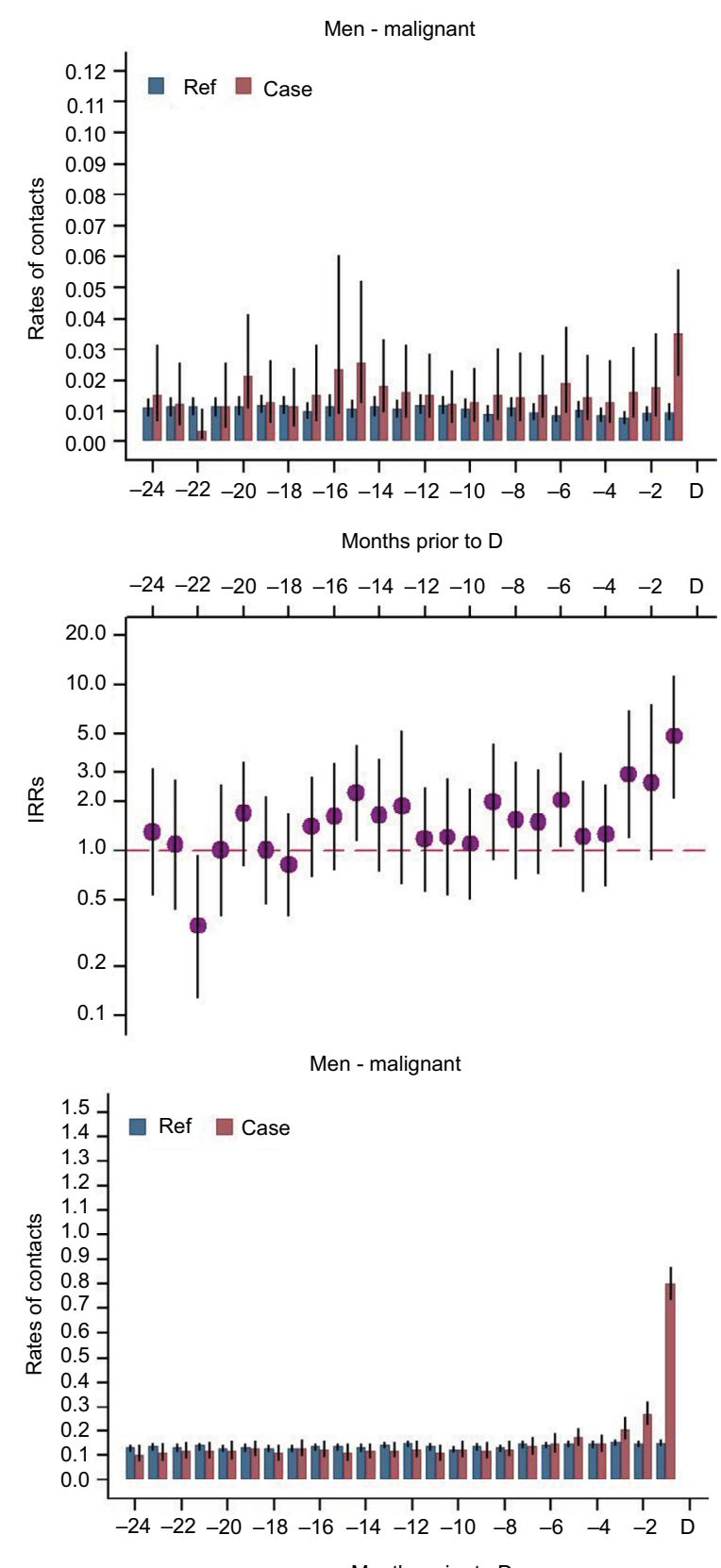

Months prior to D

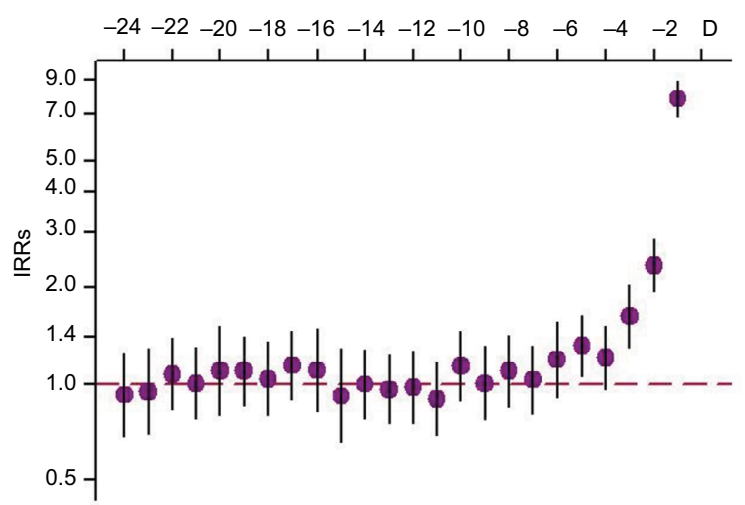

Figure S2 Total health care contacts to psychiatry $(\mathbf{A})$ and other hospital contacts $(\mathbf{B})$ among patients with malignant primary intracranial tumors.

Notes: Upper parts of $\mathbf{A}$ and $\mathbf{B}$ : average rates of contacts for patients and comparison subjects 24 months prior to diagnosis/index date. Lower parts of $\mathbf{A}$ and $\mathbf{B}$ : IRRs with $95 \%$ Cls.

Abbreviations: $\mathrm{Cl}$, confidence interval; D, date of diagnosis/index date; IRR, incidence rate ratio; Ref, reference. 
Table SI The associations between health care contacts and being diagnosed with a primary intracranial tumor

\begin{tabular}{|c|c|c|c|c|c|c|c|c|c|c|c|c|}
\hline \multicolumn{13}{|c|}{ Benign tumors } \\
\hline \multirow{3}{*}{$\begin{array}{l}\text { Number of } \\
\text { contacts by } \\
\text { specialty }\end{array}$} & \multicolumn{6}{|c|}{ Women } & \multicolumn{6}{|c|}{ Men } \\
\hline & \multicolumn{2}{|c|}{ Patients } & \multicolumn{2}{|c|}{$\begin{array}{l}\text { Comparison } \\
\text { subjects }\end{array}$} & \multirow[t]{2}{*}{ OR } & \multirow[t]{2}{*}{$95 \% \mathrm{Cl}$} & \multicolumn{2}{|c|}{ Patients } & \multicolumn{2}{|c|}{$\begin{array}{l}\text { Comparison } \\
\text { subjects }\end{array}$} & \multirow[t]{2}{*}{ OR } & \multirow[t]{2}{*}{$95 \% \mathrm{Cl}$} \\
\hline & $\mathbf{n}$ & $\%$ & $n$ & $\%$ & & & $\mathbf{n}$ & $\%$ & $\bar{n}$ & $\%$ & & \\
\hline \multicolumn{13}{|l|}{ ENT } \\
\hline 0 & $|, 79|$ & 80.8 & 19,677 & 88.8 & 0.54 & $0.48-0.61$ & $\mathrm{I}, 157$ & 80.5 & 12,826 & 89.2 & 0.52 & $0.45-0.61$ \\
\hline $\mathrm{I}-2$ & 279 & 12.6 & 1,816 & 8.2 & 1.57 & $1.36-1.81$ & 177 & 12.3 & 1,120 & 7.8 & 1.57 & $1.32-1.88$ \\
\hline$\geq 3$ & 146 & 6.6 & 667 & 3.0 & 2.13 & $|.74-2.6|$ & 104 & 7.2 & 434 & 3.0 & 2.29 & $1.79-2.95$ \\
\hline \multicolumn{13}{|c|}{ Ophthalmology } \\
\hline 0 & $\mathrm{I}, 684$ & 76.0 & 17,717 & 80 & 0.82 & $0.73-0.91$ & $\mathrm{I}, 153$ & 80.2 & 12,210 & 84.9 & 0.69 & $0.60-0.81$ \\
\hline $1-2$ & 368 & 16.6 & 3,256 & 14.7 & 1.12 & $0.99-1.27$ & 215 & 15.0 & 1,593 & II.I & 1.44 & $1.22-1.70$ \\
\hline$\geq 3$ & 164 & 7.4 & $\mathrm{I}, 187$ & 5.4 & 1.37 & $1.14-1.64$ & 70 & 4.9 & 577 & 4.0 & 1.22 & $0.92-1.60$ \\
\hline \multicolumn{13}{|l|}{ Neurology } \\
\hline 0 & 2,053 & 92.6 & 21,299 & 96.1 & 0.58 & $0.48-0.71$ & I,359 & 94.5 & 13,842 & 96.3 & 0.78 & $0.59-1.03$ \\
\hline $\mathrm{I}-2$ & 102 & 4.6 & 652 & 2.9 & 1.42 & $1.12-1.80$ & 59 & 4.1 & 395 & 2.7 & 1.32 & $0.98-1.80$ \\
\hline$\geq 3$ & 61 & 2.8 & 209 & 0.9 & 2.39 & $1.66-3.44$ & 20 & 1.4 & 143 & 1.0 & 1.14 & $0.66-1.97$ \\
\hline \multicolumn{13}{|l|}{ Psychiatry } \\
\hline 0 & 2,137 & 96.4 & 21,444 & 96.8 & 1.06 & $0.81-1.39$ & $\mathrm{I}, 394$ & 96.9 & 14,056 & 97.7 & 0.70 & $0.48-1.01$ \\
\hline $\mathrm{I}-2$ & 19 & 0.9 & 216 & 1.0 & 0.72 & $0.43-1.21$ & 19 & 1.3 & 100 & 0.7 & 2.04 & $1.16-3.57$ \\
\hline$\geq 3$ & 60 & 2.7 & 500 & 2.3 & 1.05 & $0.77-1.43$ & 25 & 1.7 & 224 & 1.6 & 1.16 & $0.72-1.88$ \\
\hline \multicolumn{13}{|c|}{ Malignant tumors } \\
\hline \multirow{4}{*}{$\begin{array}{l}\text { Number of } \\
\text { contacts by } \\
\text { specialty }\end{array}$} & \multicolumn{6}{|c|}{ Women } & \multicolumn{6}{|c|}{ Men } \\
\hline & \multirow{2}{*}{\multicolumn{2}{|c|}{ Patients }} & \multirow{2}{*}{\multicolumn{2}{|c|}{$\begin{array}{l}\text { Comparison } \\
\text { subjects }\end{array}$}} & OR & $95 \% \mathrm{Cl}$ & Patie & & Compa & ison & OR & $95 \% \mathrm{Cl}$ \\
\hline & & & & & & & & & subject & & & \\
\hline & $\mathbf{n}$ & $\%$ & $\mathbf{n}$ & $\%$ & & & $\mathbf{n}$ & $\%$ & $\mathbf{n}$ & $\%$ & & \\
\hline ENT & & & & & & & & & & & & \\
\hline 0 & 816 & 85.8 & 8,315 & 87.4 & 0.92 & $0.75-1.13$ & 1,169 & 88.5 & ||$, 64 \mid$ & 88.1 & 1.14 & $0.94-1.38$ \\
\hline $\mathrm{I}-2$ & 99 & 10.4 & 902 & 9.5 & 1.06 & $0.84-1.33$ & 103 & 7.8 & $|| 2 \mid$, & 8.5 & 0.84 & $0.68-1.05$ \\
\hline$\geq 3$ & 36 & 3.8 & 293 & 3.1 & I.II & $0.75-1.63$ & 49 & 3.7 & 448 & 3.4 & 0.95 & $0.68-1.33$ \\
\hline Ophthalmolog & & & & & & & & & & & & \\
\hline 0 & 727 & 76.4 & 7,357 & 77.4 & 0.92 & $0.78-1.08$ & 1,099 & 83.2 & 10,924 & 82.7 & 1.01 & $0.87-1.19$ \\
\hline $1-2$ & 159 & 16.7 & $\mathrm{I}, 547$ & 16.3 & 1.05 & $0.87-1.26$ & 149 & 11.3 & 1,682 & 12.7 & 0.90 & $0.75-1.08$ \\
\hline$\geq 3$ & 65 & 6.8 & 606 & 6.4 & 1.18 & $0.90-1.56$ & 73 & 5.5 & 604 & 4.6 & 1.27 & $0.98-1.66$ \\
\hline Neurology & & & & & & & & & & & & \\
\hline 0 & 903 & 95.0 & 9,123 & 95.9 & 0.87 & $0.63-1.20$ & 1,253 & 94.9 & 12,695 & 96.1 & 0.77 & $0.59-1.02$ \\
\hline $\mathrm{I}-2$ & 30 & 3.2 & 296 & 3.1 & 0.82 & $0.56-1.21$ & 54 & 4.1 & 377 & 2.9 & $\mathrm{I} .4 \mathrm{I}$ & $1.05-1.91$ \\
\hline$\geq 3$ & 18 & 1.9 & 91 & 1.0 & 2.44 & $1.42-4.19$ & 14 & I.I & 138 & 1.0 & 0.98 & $0.55-1.76$ \\
\hline Psychiatry & & & & & & & & & & & & \\
\hline 0 & 922 & 97.0 & 9,225 & 97.0 & 0.97 & $0.64-1.48$ & I,297 & 98.2 & 12,936 & 97.9 & 1.08 & $0.67-1.74$ \\
\hline $1-2$ & 7 & 0.7 & 94 & 1.0 & 0.72 & $0.3|-1.7|$ & $<5$ & - & 96 & 0.7 & 0.48 & $0.18-1.31$ \\
\hline$\geq 3$ & 22 & 2.3 & 191 & 2.0 & 1.15 & $0.70-1.88$ & 20 & 1.5 & 178 & 1.3 & 1.16 & $0.67-2.00$ \\
\hline
\end{tabular}

Notes: OR adjusted for known specialty-specific disease. Adjusted for country of origin, $\mathrm{CCl}$, marital status, income, labor market affiliation, highest educational level, and known specialty-specific disease.

Abbreviations: $\mathrm{CCl}$, Charlson Comorbidity Index; $\mathrm{Cl}$, confidence interval; ENT, ear-nose-throat; OR, odds ratio. 
Table S2 Proportion of patients and comparison subjects with known disease 10 years before study entry (before 2 years prior to date of diagnosis/index date)

\begin{tabular}{|c|c|c|c|c|}
\hline \multicolumn{5}{|l|}{ Benign tumors } \\
\hline \multirow[t]{2}{*}{ Disease category } & \multicolumn{2}{|c|}{ Patients with disease } & \multicolumn{2}{|c|}{ Comparison subjects } \\
\hline & $\%$ & $\mathbf{n}$ & $\%$ & $\mathbf{n}$ \\
\hline Headache and migraine $^{a}$ & 1.7 & 62 & 1.2 & 426 \\
\hline Epilepsyb & 2.1 & 75 & 0.9 & 327 \\
\hline ENT disease ${ }^{c}$ & 11.4 & 417 & 7.6 & 2,789 \\
\hline Ophthalmologic disease ${ }^{d}$ & 12.9 & 470 & 10.6 & 3,869 \\
\hline Neurological disease excluding epilepsy, etc. ${ }^{e}$ & 9.7 & 353 & 6.4 & 2,351 \\
\hline Psychiatric disease excluding dementia ${ }^{f}$ & 5.4 & 198 & 4.4 & 1,596 \\
\hline Neurological disease ${ }^{g}$ & 11.1 & 407 & 7.1 & 2,607 \\
\hline \multicolumn{5}{|l|}{ Malignant tumors } \\
\hline \multirow[t]{2}{*}{ Disease category } & \multicolumn{2}{|c|}{ Patients with disease } & \multicolumn{2}{|c|}{ Comparison subjects } \\
\hline & $\%$ & $\mathbf{n}$ & $\%$ & $\mathbf{n}$ \\
\hline Headache and migraine ${ }^{a}$ & 0.5 & 11 & 0.9 & 212 \\
\hline Epilepsy ${ }^{b}$ & 2.0 & 45 & 0.9 & 208 \\
\hline ENT disease ${ }^{c}$ & 11.8 & 268 & 9.6 & 2,177 \\
\hline Ophthalmologic disease ${ }^{d}$ & 11.0 & 249 & 11.7 & 2,656 \\
\hline Neurological disease excluding epilepsy, etc. ${ }^{e}$ & 6.6 & 150 & 6.5 & $\mathrm{I}, 48 \mathrm{I}$ \\
\hline Psychiatric disease excluding dementia ${ }^{f}$ & 4.3 & 97 & 4.3 & 968 \\
\hline Neurological disease ${ }^{g}$ & 8.1 & 184 & 7.2 & 1,639 \\
\hline
\end{tabular}

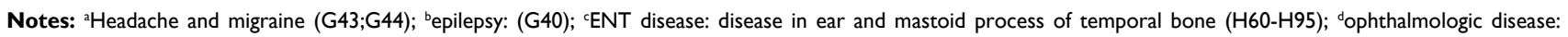

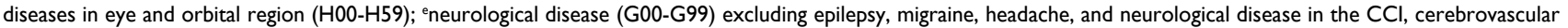
disease (G45;G46), hemiplegia (G81;G82), dementia (G30); ' 'psychiatric disease excluding dementia, psychiatric disease, and behavioral disorders (F03-F05.0 and F05.2-F99) excluding F00-F03; F05.I; gneurological disease excluding neurological disease in the $\mathrm{CCI}$.

Abbreviations: $\mathrm{CCl}$, Charlson Comorbidity Index; ENT, ear-nose-throat.

Clinical Epidemiology is an international, peer-reviewed, open access, online journal focusing on disease and drug epidemiology, identification of risk factors and screening procedures to develop optimal preventative initiatives and programs. Specific topics include: diagnosis, prognosis, treatment, screening, prevention, risk factor modification,

\section{Dovepress}

systematic reviews, risk and safety of medical interventions, epidemiology and biostatistical methods, and evaluation of guidelines, translational medicine, health policies and economic evaluations. The manuscript management system is completely online and includes a very quick and fair peer-review system, which is all easy to use. 